KYUNGPOOK Math. J. 53(2013), 49-86

http://dx.doi.org/10.5666/KMJ.2013.53.1.49

\title{
A Note on Maass-Jacobi Forms II
}

\author{
JAE-HYUN YANG \\ Department of Mathematics, Inha University, Incheon 402-751, Korea \\ e-mail : jhyang@inha.ac.kr
}

Abstract. This article is a continuation of the paper [21]. In this paper we deal with Maass-Jacobi forms on the Siegel-Jacobi space $\mathbb{H} \times \mathbb{C}^{m}$, where $\mathbb{H}$ denotes the Poincaré upper half plane and $m$ is any positive integer.

\section{Introduction}

This article is a continuation of the paper [21]. Recently A. Pitale [14], K. Bringmann and O. Richter [4], and C. Conley and M. Raum [5] defined another notion of Maass-Jacobi forms and studied some properties of Maass-Jacobi forms. In [4], [14] and [21], the authors considered the case $n=m=1$ and in [5], the authors dealt with the case $n=1$ and $m$ is arbitrary. In this paper, we consider mainly the case $n=1$ and $m$ is an arbitrary positive integer.

This paper is organized as follows. In Section 2, we give some useful geometric properties of the Siegel-Jacobi space $\mathbb{H} \times \mathbb{C}^{m}$. We study the invariant metrics, their Laplacians, a fundamental domain, geodesics, the scalar curvature and invariant differential forms on $\mathbb{H} \times \mathbb{C}^{m}$. In Section 3 we describe the center of the universal enveloping algebra of the complexfied Jacobi Lie algebra. This work is due to Conley and Raum [5]. In Section 4, we present some interesting and important results on invariant differential operators on the Siegel-Jacobi space $\mathbb{H} \times \mathbb{C}^{m}$. In Section 5 , we discuss the notion of Maass-Jacobi forms introduced by J.-H. Yang [21]. MaassJacobi forms play an important role in the spectral theory of the Laplace operator on a fundamental domain for the Siegel-Jacobi space $\mathbb{H} \times \mathbb{C}^{m}$. In Section 6 , we discuss the notion of Maass-Jacobi forms introduced by A. Pitale [14], BringmanRichter [4] and Conley-Raum [5]. We describe the results obtained in [4] and [5]. More precisely the authors of [4] and [5] obtained an explicit Fourier expansion of

Received August 23, 2011; accepted September 17, 2012. 2010 Mathematics Subject Classification: 11F50, 11F30.

Key words and phrases: Maass-Jacobi forms, invariant differential operators, fundamental domains, Casimir operators, skew-holomorphic Jacobi forms, covariant differential operators.

This work was supported by Basic Science Program through the National Research Foundation of Korea(NRF) funded by the Ministry of Education, Science and Technology (45294-01). 
the Poincaré series that is an example of harmonic Maass-Jacobi form. In Section 7, we discuss skew-holomorphic Jacobi forms introduced by N.-P. Skoruppa [18]. We describe the relation between cuspidal harmonic Maass-Jacobi forms and cuspidal skew-holomorphic Jacobi forms via the lowering operator $D_{-}^{(\mathcal{M})}$ (cf. (7.3)) In Section 8 , we briefly review some results on covariant differential operators on the SiegelJacobi space $\mathbb{H} \times \mathbb{C}^{m}$ obtained by Conley and Raum [5]. In the final section we briefly mention two notions of Maass-Jacobi forms on the Siegel-Jacobi space $\mathbb{H}_{n} \times \mathbb{C}^{(m, n)}$ for the general case $n>1$ and $m>1$. Here $\mathbb{H}_{n}$ denotes the Siegel upper half plane of degree $n$. We present some natural problems related to the study of Maass-Jacobi forms.

Notations: We denote by $\mathbb{Q}, \mathbb{R}$ and $\mathbb{C}$ the field of rational numbers, the field of real numbers and the field of complex numbers respectively. We denote by $\mathbb{Z}$ and $\mathbb{Z}^{+}$the ring of integers and the set of all positive integers respectively. $\mathbb{R}^{\times}$denotes the set of all nonzero real numbers. The symbol ":=" means that the expression on the right is the definition of that on the left. For two positive integers $k$ and $l$, $F^{(k, l)}$ denotes the set of all $k \times l$ matrices with entries in a commutative ring $F$. For a square matrix $A \in F^{(k, k)}$ of degree $k, \operatorname{tr}(A)$ denotes the trace of $A$. For any $M \in F^{(k, l)},{ }^{t} M$ denotes the transpose matrix of $M$. For $A \in F^{(k, l)}$ and $B \in F^{(k, k)}$, we set $B[A]={ }^{t} A B A$. For a complex matrix $A, \bar{A}$ denotes the complex conjugate of $A$. For $A \in \mathbb{C}^{(k, l)}$ and $B \in \mathbb{C}^{(k, k)}$, we use the abbreviation $B\{A\}={ }^{t} \bar{A} B A$. For a positive integer $n, I_{n}$ denotes the identity matrix of degree $n$. For a positive integer $m$ and a commutative ring $F$, we denote by $S(m, F)$ the space of all $m \times m$ symmetric matrices with entries in $F$. For a complex number $z,|z|$ denotes the absolute value of $z$. For a complex number $z, \operatorname{Re} z$ and $\operatorname{Im} z$ denote the real part of $z$ and the imaginary part of $z$ respectively.

\section{Geometric properties of the Siegel-Jacobi space $\mathbb{H} \times \mathbb{C}^{m}$}

We fix a positive integer $m$ throughout this paper and let

$$
\mathbb{H}=\{\tau \in \mathbb{C} \mid \operatorname{Im} \tau>0\}
$$

be the Poincaré upper half plane. Let $G=S L_{2}(\mathbb{R})$ be the special linear group of degree 2 and let

$$
H_{\mathbb{R}}^{(m)}=\left\{(\lambda, \mu ; \kappa) \mid \lambda, \mu \in \mathbb{R}^{m}, \kappa \in \mathbb{R}^{(m, m)}, \kappa+\mu^{t} \lambda \text { symmetric }\right\}
$$

be the Heisenberg group endowed with the following multiplication law

$$
(\lambda, \mu ; \kappa) \circ\left(\lambda^{\prime}, \mu^{\prime} ; \kappa^{\prime}\right)=\left(\lambda+\lambda^{\prime}, \mu+\mu^{\prime} ; \kappa+\kappa^{\prime}+\lambda^{t} \mu^{\prime}-\mu^{t} \lambda^{\prime}\right)
$$

with $(\lambda, \mu ; \kappa),\left(\lambda^{\prime}, \mu^{\prime} ; \kappa^{\prime}\right) \in H_{\mathbb{R}}^{(m)}$. We define the semidirect product of $S L_{2}(\mathbb{R})$ and $H_{\mathbb{R}}^{(m)}$

$$
G^{J}=S L_{2}(\mathbb{R}) \ltimes H_{\mathbb{R}}^{(m)}
$$


endowed with the following multiplication law

$$
(M,(\lambda, \mu ; \kappa)) \cdot\left(M^{\prime},\left(\lambda^{\prime}, \mu^{\prime} ; \kappa^{\prime}\right)\right)=\left(M M^{\prime},\left(\tilde{\lambda}+\lambda^{\prime}, \tilde{\mu}+\mu^{\prime} ; \kappa+\kappa^{\prime}+\tilde{\lambda}^{t} \mu^{\prime}-\tilde{\mu}^{t} \lambda^{\prime}\right)\right)
$$

with $M, M^{\prime} \in S L_{2}(\mathbb{R}),(\lambda, \mu ; \kappa),\left(\lambda^{\prime}, \mu^{\prime} ; \kappa^{\prime}\right) \in H_{\mathbb{R}}^{(m)}$ and $(\tilde{\lambda}, \tilde{\mu})=(\lambda, \mu) M^{\prime}$. Then $G^{J}$ acts on the Siegel-Jacobi space $\mathbb{H} \times \mathbb{C}^{m}$ of degree 1 and index $m$ transitively by

$$
(M,(\lambda, \mu ; \kappa)) \cdot(\tau, z)=\left((a \tau+b)(c \tau+d)^{-1},(z+\lambda \tau+\mu)(c \tau+d)^{-1}\right),
$$

where $M=\left(\begin{array}{ll}a & b \\ c & d\end{array}\right) \in S L_{2}(\mathbb{R}),(\lambda, \mu ; \kappa) \in H_{\mathbb{R}}^{(m)}, \tau \in \mathbb{H}$ and $z={ }^{t}\left(z_{1}, z_{2}, \cdots, z_{m}\right) \in$ $\mathbb{C}^{m}$ with $z_{i} \in \mathbb{C}(1 \leq i \leq m)$. We note that the Jacobi group $G^{J}$ is not a reductive Lie group and that the homogeneous space $\mathbb{H} \times \mathbb{C}^{m}$ is not a symmetric space.

For a coordinate $(\tau, z) \in \mathbb{H} \times \mathbb{C}^{n}$, we write $\tau=x+i y$ with $x$ real and $y>0$, and

$$
z={ }^{t}\left(z_{1}, z_{2}, \cdots, z_{m}\right), \quad z_{j}=u_{j}+i v_{j}, \quad u_{j}, v_{j} \text { real, } \quad i=1,2, \cdots, m .
$$

According to [23], for any two positive real numbers $A$ and $B$, the following metric given by

$$
\begin{aligned}
d s_{m ; A, B}^{2}= & \frac{1}{y^{3}}\left(A y+B \sum_{j=1}^{m} v_{j}^{2}\right) d \tau d \bar{\tau} \\
& +\frac{B}{y^{2}}\left\{y \sum_{j=1}^{m} d z_{j} d \bar{z}_{j}-\sum_{j=1}^{m} v_{j}\left(d \tau d \bar{z}_{j}+d \bar{\tau} d \bar{z}_{j}\right)\right\} \\
= & \frac{1}{y^{3}}\left(A y+B \sum_{j=1}^{m} v_{j}^{2}\right)\left(d x^{2}+d y^{2}\right) \\
& +\frac{B}{y^{2}}\left\{y \sum_{j=1}^{m}\left(d u_{j}^{2}+d v_{j}^{2}\right)-2 \sum_{j=1}^{m} v_{j}\left(d x d u_{j}+d y d v_{j}\right)\right\}
\end{aligned}
$$

is a Kähler metric on $\mathbb{H} \times \mathbb{C}^{m}$ invariant under the action (2.1) of $G^{J}$.

We put

$$
M_{1}:=\operatorname{tr}\left(y \frac{\partial^{t}}{\partial z}\left(\frac{\partial}{\partial \bar{z}}\right)\right)=y \sum_{j=1}^{m} \frac{\partial^{2}}{\partial z_{j} \partial \bar{z}_{j}}=\frac{y}{4}\left(\frac{\partial}{\partial u_{j}^{2}}+\frac{\partial}{\partial v_{j}^{2}}\right)
$$


and

$$
\begin{aligned}
(2.4) M_{2}:= & y^{2} \frac{\partial^{2}}{\partial \tau \partial \bar{\tau}}+\sum_{a, b=1}^{m} v_{a} v_{b} \frac{\partial^{2}}{\partial z_{a} \partial \bar{z}_{b}}+y \sum_{j=1}^{m} v_{j}\left(\frac{\partial^{2}}{\partial \tau \partial \bar{z}_{j}}+\frac{\partial^{2}}{\partial \bar{\tau} \partial z_{j}}\right) \\
= & \frac{1}{4}\left\{y^{2}\left(\frac{\partial^{2}}{\partial x^{2}}+\frac{\partial^{2}}{\partial y^{2}}\right)+\sum_{a=1}^{m} v_{a}^{2}\left(\frac{\partial^{2}}{\partial u_{a}^{2}}+\frac{\partial^{2}}{\partial v_{a}^{2}}\right)\right\} \\
& +\frac{1}{2} \sum_{1 \leq a<b \leq m} v_{a} v_{b}\left(\frac{\partial^{2}}{\partial u_{a} \partial u_{b}}+\frac{\partial^{2}}{\partial v_{a} \partial v_{b}}\right) . \\
& +\frac{y}{2} \sum_{j=1}^{m} v_{j}\left(\frac{\partial^{2}}{\partial x \partial u_{j}}+\frac{\partial^{2}}{\partial y \partial v_{j}}\right) .
\end{aligned}
$$

Then $M_{1}$ and $M_{2}$ are differential operators on $\mathbb{H} \times \mathbb{C}^{m}$ invariant under the action (2.1). The author [23] proved that

$$
\Delta_{m ; A, B}:=\frac{4}{B} M_{1}+\frac{4}{A} M_{2}
$$

is the Laplacian of $\left(\mathbb{H} \times \mathbb{C}^{m}, d s_{m ; A, B}^{2}\right)$. Furthermore the following $2(m+1)$-differential form

$$
d v=d x \wedge d y \wedge d u_{1} \wedge \cdots \wedge d u_{m} \wedge d v_{1} \wedge \cdots \wedge d v_{m}
$$

is a $G^{J}$-invariant volume element on the Siegel-Jacobi space $\mathbb{H} \times \mathbb{C}^{m}$.

Let $K^{J}$ be the stabilizer of $G^{J}$ at $(i, 0)$. Then

$$
K^{J}=\left\{\left(\left(\begin{array}{cc}
a & -b \\
b & a
\end{array}\right),(0,0, R)\right) \mid a^{2}+b^{2}=1, a, b \in \mathbb{R}, R={ }^{t} R \in \mathbb{R}^{(m, m)}\right\} .
$$

Thus $G^{J} / K^{J}$ is diffeomorphic to $\mathbb{H} \times \mathbb{C}^{m}$ via

$$
g K^{J} \longmapsto g \cdot(i, 0)=\left(\frac{a i+b}{c i+d}, \frac{\lambda i+\mu}{c i+d}\right),
$$

where $\left(\begin{array}{ll}a & b \\ c & d\end{array}\right) \in S L_{2}(\mathbb{R})$ and $(\lambda, \mu ; \kappa) \in H_{\mathbb{R}}^{(m)}$. The Siegel-Jacobi space $\mathbb{H} \times \mathbb{C}^{m}$ is a homogeneous space which is not symmetric. Let $\mathfrak{k}^{J}$ be the Lie algebra of $K^{J}$. Then the Lie algebra $\mathfrak{g}^{J}$ of $G^{J}$ has the Cartan decomposition

$$
\mathfrak{g}^{J}=\mathfrak{k}^{J}+\mathfrak{p}^{J},
$$

where

$$
\begin{aligned}
\mathfrak{g}^{J} & =\left\{\left(\left(\begin{array}{cc}
x & y \\
z & -x
\end{array}\right),(P, Q, R)\right) \mid x, y, z \in \mathbb{R}, P, Q \in \mathbb{R}^{m}, R={ }^{t} R \in \mathbb{R}^{(m, m)}\right\}, \\
\mathfrak{k}^{J} & =\left\{\left(\left(\begin{array}{cc}
0 & x \\
-x & 0
\end{array}\right),(0,0, R)\right) \mid x \in \mathbb{R}, R={ }^{t} R \in \mathbb{R}^{(m, m)}\right\}, \\
\mathfrak{p}^{J} & =\left\{\left(\left(\begin{array}{cc}
x & y \\
y & -x
\end{array}\right),(P, Q, 0)\right) \mid x, y \in \mathbb{R}, P, Q \in \mathbb{R}^{m}\right\} .
\end{aligned}
$$


Lemma 2.1. We have the relations

$$
\left[\mathfrak{k}^{J}, \mathfrak{k}^{J}\right] \subset \mathfrak{k}^{J} \quad \text { and } \quad\left[\mathfrak{k}^{J}, \mathfrak{p}^{J}\right] \subset \mathfrak{p}^{J} .
$$

Proof. The Lie bracket operation on $\mathfrak{g}^{J}$ is given by

$$
\left[\left(X_{1},\left(P_{1}, Q_{1}, R_{1}\right)\right),\left(X_{2},\left(P_{2}, Q_{2}, R_{2}\right)\right)\right]=\left(X^{*},\left(P^{*}, Q^{*}, R^{*}\right)\right),
$$

where $X_{1}, X_{2} \in \mathfrak{s l}_{2}(\mathbb{R}), P_{1}, Q_{1}, P_{2}, Q_{2} \in \mathbb{R}^{m}, R_{1}={ }^{t} R_{1}, R_{2}={ }^{t} R_{2} \in \mathbb{R}^{(m, m)}$,

$$
\begin{aligned}
X^{*} & =\left[X_{1}, X_{2}\right]=X_{1} X_{2}-X_{2} X_{1}, \\
\left(P^{*}, Q^{*}\right) & =\left(P_{1}, Q_{1}\right) X_{2}-\left(P_{2}, Q_{2}\right) X_{1}, \\
R^{*} & =P_{1}{ }^{t} Q_{2}-P_{2}{ }^{t} Q_{1}+Q_{2}{ }^{t} P_{1}-Q_{1}{ }^{t} P_{2} .
\end{aligned}
$$

The relations (2.8) follow immediately from Formula (2.9).

Remark 2.1. The relation

$$
\left[\mathfrak{p}^{J}, \mathfrak{p}^{J}\right] \subset \mathfrak{k}^{J}
$$

does not hold.

The vector space $\mathfrak{p}^{J}$ can be regarded as the tangent space of the Siegel-Jacobi space $\mathbb{H} \times \mathbb{C}^{m} \cong G^{J} / K^{J}$ at $(i, 0)$. We define a complex structure $I^{J}$ on the tangent space $\mathfrak{p}^{J}$ of $\mathbb{H} \times \mathbb{C}^{m} \cong G^{J} / K^{J}$ at $(i, 0)$ by

$$
I^{J}\left(\left(\begin{array}{cc}
x & y \\
y & -x
\end{array}\right),(P, Q, 0)\right)=\left(\left(\begin{array}{cc}
y & -x \\
-x & -y
\end{array}\right),(Q,-P, 0)\right) .
$$

Let

$$
\mathfrak{p}=\left\{\left(\begin{array}{cc}
x & y \\
y & -x
\end{array}\right) \in \mathbb{R}^{(2,2)} \mid x, y \in \mathbb{R}\right\}
$$

be the real vector space of dimension 2 . Identifying $\mathfrak{p}$ with $\mathbb{C}$ via

$$
\left(\begin{array}{cc}
x & y \\
y & -x
\end{array}\right) \longmapsto x+i y \in \mathbb{C}
$$

and identifying $\mathbb{R}^{m} \times \mathbb{R}^{m}$ with $\mathbb{C}^{m}$ via

$$
(P, Q) \longmapsto Q+i P, \quad P, Q \in \mathbb{R}^{m},
$$

we may regard the complex structure $I^{J}$ as a real linear map on $\mathbb{C} \times \mathbb{C}^{m}$ defined by

$$
I^{J}(x+i y, Q+i P)=(-y+i x,-P+i Q), \quad x+i y \in \mathbb{C}, Q+i P \in \mathbb{C}^{m} .
$$

Clearly $I^{J}$ extends complex linearly on the complexification $\mathfrak{p}_{\mathbb{C}}^{J}=\mathfrak{p}^{J} \otimes_{\mathbb{R}} \mathbb{C}$ of $\mathfrak{p}^{J}$. Then $\mathfrak{p}_{\mathbb{C}}^{J}$ has a decomposition

$$
\mathfrak{p}_{\mathbb{C}}^{J}=\mathfrak{p}_{+}^{J} \oplus \mathfrak{p}_{-}^{J}
$$


where $\mathfrak{p}_{+}^{J}$ (resp. $\left.\mathfrak{p}_{-}^{J}\right)$ denotes the $(+i)$-eigenspace (resp. (-i)-eigenspace) of $I^{J}$. Precisely, both $\mathfrak{p}_{+}^{J}$ and $\mathfrak{p}_{-}^{J}$ are given by

$$
\mathfrak{p}_{+}^{J}=\left\{\left(\left(\begin{array}{cc}
x & i x \\
i x & -x
\end{array}\right),(P, i P, 0)\right) \mid x \in \mathbb{C}, P \in \mathbb{C}^{m}\right\}
$$

and

$$
\mathfrak{p}_{-}^{J}=\left\{\left(\left(\begin{array}{cc}
x & -i x \\
-i x & -x
\end{array}\right),(P,-i P, 0)\right) \mid x \in \mathbb{C}, P \in \mathbb{C}^{m}\right\}
$$

Proposition 2.1. Fix an element $g=(M,(\lambda, \mu ; \kappa)) \in G^{J}$ with $M=\left(\begin{array}{ll}a & b \\ c & d\end{array}\right) \in$ $S L_{2}(\mathbb{R})$ and $(\lambda, \mu ; \kappa) \in H_{\mathbb{R}}^{(m)}$. We let $\left(\tau_{*}, z_{*}\right)=g \cdot(\tau, z)$. Let

$$
\mathbb{F}_{g}: \mathbb{H} \times \mathbb{C}^{m} \longrightarrow \mathbb{H} \times \mathbb{C}^{m}
$$

be the biholomorphic mapping defined by the action (2.1) of $g$. Then the differential mapping

$$
d \mathbb{F}_{g}: T_{(\tau, z)}\left(\mathbb{H} \times \mathbb{C}^{m}\right) \longrightarrow T_{\left(\tau_{*}, z_{*}\right)}\left(\mathbb{H} \times \mathbb{C}^{m}\right)
$$

is given by

$$
(w, \xi) \longmapsto(w(g), \xi(g)), \quad w \in \mathbb{C}, \xi \in \mathbb{C}^{m}
$$

with

$$
w(g)=\frac{w}{(c \tau+d)^{2}} \quad \text { and } \quad \xi(g)=\frac{\xi}{c \tau+d}+\frac{w(d \lambda-c \mu-c z)}{(c \tau+d)^{2}} .
$$

Here we identified $\mathfrak{p}^{J}$ with $\mathbb{C} \times \mathbb{C}^{m}$.

Proof. Let $\alpha(t)=(\tau(t), z(t))(-\epsilon<t<\epsilon, \epsilon>0)$ be a smooth curve in $\mathbb{H} \times \mathbb{C}^{m}$ passing through $\alpha(0)=(\tau, z)$ with $\alpha^{\prime}(0)=(w, \xi) \in T_{(\tau, z)}\left(\mathbb{H} \times \mathbb{C}^{m}\right)$. Then

$$
\begin{aligned}
\chi(t): & =g \cdot \alpha(t)=(\tau(g ; t), z(g ; t)) \\
& =\left(\frac{a \tau(t)+b}{c \tau(t)+d}, \frac{z(t)+\lambda \tau(t)+\mu}{c \tau(t)+d}\right)
\end{aligned}
$$

is a smooth curve in $\mathbb{H} \times \mathbb{C}^{m}$ passing through $\chi(0)=\left(\tau_{*}, z_{*}\right)$. Then by an easy computation, we see that

$$
\tau^{\prime}(g ; 0)=\left.\frac{\partial}{\partial t}\right|_{t=0} \tau(g ; t)=\frac{\tau^{\prime}(0)}{(c \tau+d)^{2}}=\frac{w}{(c \tau+d)^{2}}
$$

and

$$
z^{\prime}(g ; 0)=\left.\frac{\partial}{\partial t}\right|_{t=0} z(g ; t)=\frac{\xi}{c \tau+d}+\frac{w(d \lambda-c \mu-c z)}{(c \tau+d)^{2}} .
$$


Let $\Gamma_{1}:=S L_{2}(\mathbb{Z})$ be the elliptic modular group. We let

$$
\Gamma_{1, m}:=\Gamma_{1} \ltimes H_{\mathbb{Z}}^{(m)}
$$

be the arithmetic subgroup of $G^{J}$, where

$$
H_{\mathbb{Z}}^{(m)}:=\left\{(\lambda, \mu ; \kappa) \in H_{\mathbb{R}}^{(m)} \mid \lambda, \mu, \kappa \text { are integral }\right\}
$$

is a discrete subgroup of $H_{\mathbb{R}}^{(m)}$. Let $E_{k}:={ }^{t}(0, \cdots, 1,0, \cdots, 0)(1 \leq k \leq m)$ be the $m \times 1$ matrix with the $(k, 1)$-th entry 1 and other entries 0 . For an element $\tau \in \mathbb{H}$, we set for brevity

$$
F_{k}(\tau):=\tau E_{k}, \quad 1 \leq k \leq m
$$

Let

$$
\mathcal{F}:=\{\tau \in \mathbb{H}|| \tau|\geq 1, \quad| \operatorname{Re} \tau \mid \leq 1 / 2\}
$$

be a fundamental domain for $\Gamma_{1} \backslash \mathbb{H}$. We refer to [16], pp. 78-79 for more detail. For each $\tau \in \mathcal{F}$, we define the subset $P_{\tau}$ of $\mathbb{C}^{m}$ by

$$
P_{\tau}:=\left\{\sum_{k=1}^{m} \lambda_{k} E_{k}+\sum_{k=1}^{m} \mu_{k} F_{k}(\tau) \mid 0 \leq \lambda_{k}, \mu_{k} \leq 1\right\} .
$$

For each $\tau \in \mathcal{F}$, we define the subset $\mathcal{D}_{\tau}$ of $\mathbb{H} \times \mathbb{C}^{m}$ by

$$
\mathcal{D}_{\tau}:=\left\{(\tau, z) \in \mathbb{H} \times \mathbb{C}^{m} \mid z \in P_{\tau}\right\} .
$$

Theorem 2.1. The following subset

$$
\mathcal{F}_{[m]}:=\bigcup_{\tau \in \mathcal{F}} \mathcal{D}_{\tau}
$$

is a fundamental domain for $\Gamma_{1, m} \backslash\left(\mathbb{H} \times \mathbb{C}^{m}\right)$ with respect to the action (2.1).

Proof. Let $\left(\tau_{*}, z_{*}\right)$ be an arbitrary element of $\mathbb{H} \times \mathbb{C}^{m}$. We must find an element $(\tau, z)$ of $\mathcal{F}_{[m]}$ and $\gamma_{*}=(\gamma,(\lambda, \mu ; \kappa)) \in \Gamma_{1, m}$ with $\gamma \in \Gamma_{1}=S L_{2}(\mathbb{Z})$ such that $\gamma_{*} \cdot(\tau, z)=\left(\tau_{*}, z_{*}\right)$. Since $\mathcal{F}$ is a fundamental domain for $\Gamma_{1} \backslash \mathbb{H}$, there is an element $\gamma$ of $\Gamma_{1}$ and an element $\tau \in \mathcal{F}$ such that $\tau_{*}=\gamma \cdot \tau$. Here $\tau$ is unique up to the boundary of $\mathcal{F}$. We write

$$
\gamma=\left(\begin{array}{ll}
a & b \\
c & d
\end{array}\right) \in \Gamma_{1}=S L_{2}(\mathbb{Z})
$$

We can find $\lambda, \mu \in \mathbb{Z}^{m}$ and $z \in P_{\tau}$ satisfying the equation

$$
z+\lambda \tau+\mu=z_{*}(x \tau+d) .
$$


If we take $\gamma_{*}=(\gamma,(\lambda, \mu ; 0)) \in \Gamma_{1, m}$, we see that $\gamma_{*} \cdot(\tau, z)=\left(\tau_{*}, z_{*}\right)$. Therefore

$$
\mathbb{H} \times \mathbb{C}^{m}=\bigcup_{\gamma_{*} \in \Gamma_{1, m}} \gamma_{*} \cdot \mathcal{F}_{[m]} .
$$

Let $(\tau, z)$ and $\gamma_{*} \cdot(\tau, z)$ be two elements of $\mathcal{F}_{[m]}$ with $\gamma_{*}=(\gamma,(\lambda, \mu ; \kappa)) \in \Gamma_{1, m}$ with $\gamma \in \Gamma_{1}$. Then both $\tau$ and $\gamma \cdot \tau$ lie in $\mathcal{F}$. Therefore both of them either lie in the boundary of $\mathcal{F}$ or $\gamma= \pm I_{2}$. In the case that both $\tau$ and $\gamma \cdot \tau$ lie in the boundary of $\mathcal{F}$, both $(\tau, z)$ and $\gamma_{*} \cdot(\tau, z)$ lie in the boundary of $\mathcal{F}_{[m]}$. If $\gamma= \pm I_{2}$, we get

$$
z \in P_{\tau} \quad \text { and } \quad \pm(z+\lambda \tau+\mu) \in P_{\tau} .
$$

From the definition of $P_{\tau}$ and (2.16), we see that either $\lambda=\mu=0, \gamma \neq-I_{2}$ or both $z$ and $\pm(z+\lambda \tau+\mu)$ lie on the boundary of the parallelepiped $P_{\tau}$. Hence either both $(\tau, z)$ and $\gamma_{*} \cdot(\tau, z)$ lie in the boundary of $\mathcal{F}_{[m]}$ or $\gamma_{*}=\left(I_{2},(0,0 ; \kappa)\right) \in \Gamma_{1, m}$. Consequently $\mathcal{F}_{[m]}$ is a fundamental domain for $\Gamma_{1, m} \backslash\left(\mathbb{H} \times \mathbb{C}^{m}\right)$ with respect to the action (2.1).

Now we consider the Siegel-Jacobi space $\mathbb{H}_{1,1}:=\mathbb{H} \times \mathbb{C}$ endowed with the Riemannian metric (cf. (2.2))

$$
d s_{1 ; 1,1}^{2}=\frac{y+v^{2}}{y^{3}}\left(d x^{2}+d y^{2}\right)+\frac{1}{y}\left(d u^{2}+d v^{2}\right)-\frac{2 v}{y^{2}}(d x d u+d y d v),
$$

where $\tau=x+i y$ with $x, y>0$ real and $z=u+i v$ with $u, v$ real are coordinates in $\mathbb{H}_{1,1}$. Then

$$
E_{1}:=\frac{\partial}{\partial x}, \quad E_{2}:=\frac{\partial}{\partial y}, \quad E_{3}:=\frac{\partial}{\partial u}, \quad E_{4}:=\frac{\partial}{\partial v}
$$

form a local frame field on $\mathbb{H}_{1,1}$. Let $\Gamma_{i j}^{k}(i, j, k=1,2,3,4)$ be the Christoffel symbols for the Riemannian connection $\nabla$ determined uniquely by the Riemannian metric $d s_{1: 1,1}^{2}$. That is,

$$
\nabla_{E_{i}} E_{j}=\sum_{k=1}^{4} \Gamma_{i j}^{k} E_{k}, \quad i, j=1,2,3,4 .
$$

Lemma 2.2. For all $i, j, k=1,2,3,4, \Gamma_{i j}^{k}=\Gamma_{j i}^{k}$. The Christoffel symbols $\Gamma_{i j}^{k}$ 's $(1 \leq i, j, k \leq 4)$ are given by

$$
\begin{aligned}
& \Gamma_{11}^{2}=\frac{2 y+y^{2}}{2 y^{2}}, \quad \Gamma_{12}^{1}=\Gamma_{22}^{2}=-\frac{2 y+v^{2}}{2 y^{2}}, \\
& \Gamma_{11}^{4}=\frac{v^{3}}{2 y^{3}}, \quad \Gamma_{12}^{3}=\Gamma_{22}^{4}=-\frac{v^{3}}{2 y^{3}}, \\
& \Gamma_{14}^{1}=\Gamma_{23}^{1}=\Gamma_{24}^{2}=\Gamma_{33}^{4}=\frac{v}{2 y}, \\
& \Gamma_{13}^{2}=\Gamma_{34}^{3}=\Gamma_{44}^{4}=-\frac{v}{2 y}, \quad \Gamma_{13}^{4}=\frac{y-v^{2}}{2 y^{2}}, \\
& \Gamma_{14}^{3}=\Gamma_{23}^{3}=\Gamma_{24}^{4}=-\frac{y-v^{2}}{2 y^{2}}, \quad \Gamma_{33}^{2}=\frac{1}{2}, \quad \Gamma_{34}^{1}=\Gamma_{44}^{2}=-\frac{1}{2}
\end{aligned}
$$


and all other $\Gamma_{i j}^{k}=0$.

Proof. It is easy to prove the above lemma. We leave the proof to the reader.

Proposition 2.2. Let $\gamma(t)=(x(t)+i y(t), u(t)+i v(t))$ be a smooth curve in $\mathbb{H}_{1,1}$. For brevity we write

$$
\begin{aligned}
& \ddot{x}=\frac{d^{2} x}{d t^{2}}, \quad \ddot{y}=\frac{d^{2} y}{d t^{2}}, \quad \ddot{u}=\frac{d^{2} u}{d t^{2}}, \quad \ddot{v}=\frac{d^{2} v}{d t^{2}}, \\
& \dot{x}=\frac{d x}{d t}, \quad \dot{y}=\frac{d y}{d t}, \quad \dot{u}=\frac{d u}{d t}, \quad \dot{v}=\frac{d v}{d t} .
\end{aligned}
$$

Then the curve $\gamma(t)$ is a geodesic in $\mathbb{H}_{1,1}$ with respect to the metric $d s_{1 ; 1,1}^{2}$ if and only if it satisfies the following four differential equations

$$
\begin{gathered}
\ddot{x}-\frac{2 y+y^{2}}{2 y^{2}} \dot{x} \dot{y}+\frac{v}{y} \dot{x} \dot{v}+\frac{v}{y} \dot{y} \dot{u}-\dot{u} \dot{v}=0 \\
\ddot{y}+\frac{2 y+y^{2}}{2 y^{2}} \dot{x}^{2}-\frac{2 y+y^{2}}{2 y^{2}} \dot{y}^{2}+\frac{1}{2} \dot{u}^{2}-\frac{1}{2} \dot{v}^{2}-\frac{v}{y} \dot{x} \dot{u}+\frac{v}{y} \dot{y} \dot{v}=0 \\
\ddot{u}-\frac{v^{3}}{y^{3}} \dot{x} \dot{y}-\frac{y-v^{2}}{y^{2}} \dot{x} \dot{v}-\frac{y-v^{2}}{y^{2}} \dot{y} \dot{u}-\frac{v}{y} \dot{u} \dot{v}=0 \\
\ddot{v}+\frac{v^{3}}{2 y^{3}} \dot{x}^{2}-\frac{v^{3}}{2 y^{3}} \dot{y}^{2}+\frac{v}{2 y} \dot{u}^{2}-\frac{v}{2 y} \dot{v}^{2}+\frac{y-v^{2}}{y^{2}} \dot{x} \dot{u}-\frac{y-v^{2}}{y^{2}} \dot{y} \dot{v}=0
\end{gathered}
$$

Proof. Using Lemma 2.2 and the geodesic equations, we obtain the above equations.

Remark 2.2. If $u=v=0$, the equations (2.16)-(2.19) reduce to the following two equations

$$
\ddot{x}-\frac{2}{y} \dot{x} \dot{y}=0
$$

and

$$
\ddot{y}+\frac{1}{y} \dot{x}^{2}-\frac{1}{y} \dot{y}^{2}=0 .
$$

Thus these two equations (2.20) and (2.21) give geodesics in the Poincaré upper half plane $\mathbb{H}$ which are circles perpendicular to the $x$-axis or straight lines perpendicular to the $x$-axis. Therefore the curve $\gamma(t)=(x(t)+i y(t), 0)(-\infty<t<\infty)$ such that $\alpha(t)=x(t)+i y(t)$ is a geodesic in $\mathbb{H}$ is a geodesic in $\mathbb{H}_{1,1}$ with respect to the 
metric $d s_{1 ; 1,1}^{2}$.

Proposition 2.3. Let $\gamma(t)$ be a geodesic in $\mathbb{H}_{1,1}$ joining two points $\gamma(0)=\left(\tau_{1}, 0\right)$ and $\gamma(1)=\left(\tau_{2}, 0\right)$ such that $\gamma(t)$ is contained in the subset $\left\{(\tau, 0) \in \mathbb{H}_{1,1} \mid \tau \in \mathbb{H}\right\}$. Then the length $\rho$ of the geodesic segment between $\gamma(0)=\left(\tau_{1}, 0\right)$ and $\gamma(1)=\left(\tau_{2}, 0\right)$ is given by

$$
\rho=\log \frac{1+R^{1 / 2}}{1-R^{1 / 2}},
$$

where $R:=R\left(\tau_{1}, \tau_{2}\right)$ is the cross-ratio of $\tau_{1}$ and $\tau_{2}$ defined by

$$
R\left(\tau_{1}, \tau_{2}\right):=\frac{\tau_{1}-\tau_{2}}{\tau_{1}-\bar{\tau}_{2}} \cdot \frac{\bar{\tau}_{1}-\bar{\tau}_{2}}{\bar{\tau}_{1}-\tau_{2}}
$$

Proof. By remark 2.2, the length $\rho$ is equal to the length $\rho_{0}$ of the geodesic in $\mathbb{H}$ joining $\tau_{1}$ and $\tau_{2}$ with respect to the Poincaré metric

$$
d s^{2}=\frac{d x^{2}+d y^{2}}{y^{2}} .
$$

It is well known that $\rho_{0}$ is given by the formula (2.22). We refer to [17] for the general case.

Proposition 2.4. Let $\left(\tau_{1}, z_{1}\right)$ and $\left(\tau_{2}, z_{2}\right)$ be two points in the Siegel-Jacobi space $\mathbb{H} \times \mathbb{C}^{m}$. Then there exists an element $g=\left(\left(\begin{array}{ll}a & b \\ c & d\end{array}\right),(\lambda, \mu ; \kappa)\right) \in G^{J}$ such that

$$
g \cdot\left(\tau_{1}, z_{1}\right)=(i, 0) \quad \text { and } \quad g \cdot\left(\tau_{2}, z_{2}\right)=\left(i \delta, \frac{z_{2}+\lambda \tau_{2}+\mu}{c \tau_{2}+d}\right)
$$

with $\delta>0$. Therefore the length of the geodesic joining $\left(\tau_{1}, z_{1}\right)$ to $\left(\tau_{2}, z_{2}\right)$ with respect to the Riemannian metric $d s_{m ; A, B}^{2}$ is equal to that of the geodesic joining $(i, 0)$ to $\left(i \delta, \frac{z_{2}+\lambda \tau_{2}+\mu}{c \tau_{2}+d}\right)$ with respect to the metric $d s_{m ; A, B}^{2}$.

Proof. We see that there is an element $h=\left(\begin{array}{ll}a & b \\ c & d\end{array}\right) \in S L_{2}(\mathbb{R})$ such that

$$
h \cdot \tau_{1}=\frac{a \tau_{1}+b}{c \tau_{1}+d}=i \quad \text { and } \quad h \cdot \tau_{2}=\frac{a \tau_{2}+b}{c \tau_{2}+d}=i \delta
$$

with $\delta>0$. We take

$$
\lambda=-\frac{\operatorname{Im} z_{1}}{\operatorname{Im} \tau_{1}} \quad \text { and } \quad \mu=-\operatorname{Re} z_{1}+\frac{\operatorname{Re} \tau_{1} \cdot \operatorname{Im} z_{1}}{\operatorname{Im} \tau_{1}}
$$

We easily see that the element

$$
g=\left(\left(\begin{array}{ll}
a & b \\
c & d
\end{array}\right),(\lambda, \mu ; \kappa)\right) \in G^{J}
$$


satisfies the condition

$$
g \cdot\left(\tau_{1}, z_{1}\right)=(i, 0) \quad \text { and } \quad g \cdot\left(\tau_{2}, z_{2}\right)=\left(i \delta, \frac{z_{2}+\lambda \tau_{2}+\mu}{c \tau_{2}+d}\right)
$$

with $\delta>0$.

For each fixed element $g \in G^{J}$, according to the $G^{J}$-invariance of the metric $d s_{m ; A, B}^{2}$, the map $\mathbb{F}_{g}$ of $\mathbb{H} \times \mathbb{C}^{m}$ defined by the action (2.1) of $g$ is an isometry of $\mathbb{H} \times \mathbb{C}^{m}$ with respect to the metric $d s_{m ; A, B}^{2}$. Consequently we obtain the second statement.

Proposition 2.5. The scalar curvature $r(p)$ of the Siegel-Jacobi space $\left(\mathbb{H}_{1,1}, d s_{1 ; 1,1}^{2}\right)$ is -3 for each point $p$ of $\mathbb{H}_{1,1}$.

Proof. Using Lemma 2.2, we obtain the scalar curvature $r(p)=-3$ for each point $p$ of $\mathbb{H}_{1,1}$ by a tedious computation.

Now we study differential forms on $\mathbb{H} \times \mathbb{C}^{m}$ invariant under the action (2.1) of $\Gamma_{1, m}$.

Proposition 2.6. (a) Assume that

$$
\alpha=f(\tau, z) d \tau+\sum_{k=1}^{m} \phi_{k}(\tau, z) d z_{k}
$$

is a differential 1 -form on $\mathbb{H} \times \mathbb{C}^{m}$ invariant under the action (2.1) of $\Gamma_{1, m}$. Then the functions $f$ and $\phi_{k}(k=1,2, \cdots, m)$ satisfy the following conditions

$$
f(\gamma \cdot(\tau, z))=(c \tau+d)^{2} f(\tau, z)+(c \tau+d) \sum_{k=1}^{m}\left(c z_{k}+c \mu_{k}-d \lambda_{k}\right) \phi_{k}(\tau, z)
$$

and

$$
\phi_{k}(\gamma \cdot(\tau, z))=(c \tau+d) \phi_{k}(\tau, z), \quad k=1,2, \cdots, m
$$

for all $\gamma=\left(\left(\begin{array}{ll}a & b \\ c & d\end{array}\right),(\lambda, \mu ; \kappa)\right) \in \Gamma_{1, m}$ with $\lambda={ }^{t}\left(\lambda_{1}, \cdots, \lambda_{m}\right) \in \mathbb{Z}^{m}$ and $\mu=$ ${ }^{t}\left(\mu_{1}, \cdots, \mu_{m}\right) \in \mathbb{Z}^{m}$.

(b) Let

$$
\eta=d \tau \wedge d z_{1} \wedge d z_{2} \wedge \cdots \wedge d z_{m}
$$

be a differential $(m+1)$-form on $\mathbb{H} \times \mathbb{C}^{m}$. Assume that

$$
\theta=g(\tau, z) \eta^{\otimes \ell}, \quad \ell=1,2,3, \cdots,
$$

is a differential $\ell(m+1)$-form on $\mathbb{H} \times \mathbb{C}^{m}$ invariant under the action (2.1) of $\Gamma_{1, m}$. Then the function $g$ satisfies the following condition

$$
g(\gamma \cdot(\tau, z))=(c \tau+d)^{\ell(m+2)} g(\tau, z)
$$


for all $\gamma=\left(\left(\begin{array}{ll}a & b \\ c & d\end{array}\right),(\lambda, \mu ; \kappa)\right) \in \Gamma_{1, m}$.

(c) For $k=1,2, \cdots, m$, we let

$$
\widetilde{\omega}_{k}=(-1)^{m-k} d \tau \wedge d z_{1} \wedge \cdots \wedge d z_{k-1} \wedge \widehat{d z_{k}} \wedge d z_{k+1} \wedge \cdots \wedge d z_{m}
$$

be a differential $m$-form on $\mathbb{H} \times \mathbb{C}^{m}$. Assume that

$$
\beta=\sum_{k=1}^{m} a_{k}(\tau, z) \widetilde{\omega}_{k}+(-1)^{m} b(\tau, z) d z_{1} \wedge \cdots \wedge d z_{m}
$$

is a differential $m$-form on $\mathbb{H} \times \mathbb{C}^{m}$ invariant under the action (2.1) of $\Gamma_{1, m}$. Then the functions $a(\tau, z)$ and $b_{k}(k=1,2, \cdots, m)$ satisfy the following conditions

$(2.26) a_{k}(\gamma \cdot(\tau, z))=(c \tau+d)^{m+1} a_{k}(\tau, z)-(c \tau+d)^{m}\left(c z_{k}+c \mu_{k}-d \lambda_{k}\right) b(\tau, z)$

for $k-1,2, \cdots, m$ and

$$
b(\gamma \cdot(\tau, z))=(c \tau+d)^{m} b(\tau, z)
$$

for all $\gamma=\left(\left(\begin{array}{ll}a & b \\ c & d\end{array}\right),(\lambda, \mu ; \kappa)\right) \in \Gamma_{1, m}$ with $\lambda={ }^{t}\left(\lambda_{1}, \cdots, \lambda_{m}\right) \in \mathbb{Z}^{m}$ and $\mu=$ ${ }^{t}\left(\mu_{1}, \cdots, \mu_{m}\right) \in \mathbb{Z}^{m}$.

Proof. For $\gamma=\left(\left(\begin{array}{ll}a & b \\ c & d\end{array}\right),(\lambda, \mu ; \kappa)\right) \in \Gamma_{1, m}$ with $\lambda={ }^{t}\left(\lambda_{1}, \cdots, \lambda_{m}\right) \in \mathbb{Z}^{m}$ and $\mu={ }^{t}\left(\mu_{1}, \cdots, \mu_{m}\right) \in \mathbb{Z}^{m}$ and $(\tau, z) \in \mathbb{H} \times \mathbb{C}^{m}$ with $z={ }^{t}\left(z_{1}, \cdots, z_{m}\right) \in \mathbb{C}^{m}$, we set $\left(\tau^{*}, z^{*}\right)=\gamma \cdot(\tau, z)$. In other words,

$$
\tau^{*}=\frac{a \tau+b}{c \tau+d}, \quad z_{k}^{*}=\frac{z_{k}+\lambda_{k} \tau+\mu_{k}}{c \tau+d}, \quad k=1,2, \cdots, m .
$$

Then we have

$$
d \tau^{*}=\frac{d \tau}{(c \tau+d)^{2}}
$$

and

$$
d z_{k}^{*}=\left\{\frac{\lambda_{k}}{c \tau+d}-\frac{c\left(z_{k}+\lambda_{k} \tau+\mu_{k}\right)}{(c \tau+d)^{2}}\right\} d \tau+\frac{d z_{k}}{c \tau+d}, \quad k=1,2, \cdots, m .
$$

Using the formulas (2.28) and (2.29), we obtain the desired results (a), (b) and (c). 


\section{The center of the universal enveloping algebra of $\mathfrak{g}^{J}$}

In this section we describe the center of the universal enveloping algebra of the complexication of the Jacobi Lie algebra $\mathfrak{g}^{J}$ explicitly.

Let $\mathfrak{g}_{\mathbb{C}}^{J}$ be the complexification of the Jacobi Lie algebra $\mathfrak{g}^{J}$. We put the $2 \times 2$ matrices

$$
H=\left(\begin{array}{rr}
1 & 0 \\
0 & -1
\end{array}\right), \quad E=\left(\begin{array}{ll}
0 & 1 \\
0 & 0
\end{array}\right) \quad \text { and } \quad F=\left(\begin{array}{ll}
0 & 0 \\
1 & 0
\end{array}\right) .
$$

Then $\{H, E, F\}$ is a basis of the Lie algebra $\mathfrak{s l}_{2}(\mathbb{C})$. Let $\epsilon_{i j}(1 \leq i \leq m, j=1,2)$ be the $m \times 2$ matrices whose $(i, j)$-th entry is 1 and whose other entries are zero, and let $E_{k l}$ be the $m \times m$ elementary matrix whose $(k, l)$-th entry is 1 and whose other entries are zero. We set $e_{i}:=\epsilon_{i 1}, f_{i}:=\epsilon_{i 2}(1 \leq i \leq m)$ and

$$
R_{k l}:=\frac{1}{2}\left(E_{k l}+E_{j i}\right), \quad R_{k l}=R_{l k}, \quad 1 \leq k, l \leq m .
$$

Then $\left\{H, E, F, e_{i}, f_{i}, R_{k l} \mid 1 \leq i \leq m, 1 \leq k \leq l \leq m\right\}$ is a basis for $\mathfrak{g}_{\mathbb{C}}^{J}$. It is easily seen that

$$
z_{m}:=\left\{(0,(0,0, R)) \in \mathfrak{g}_{\mathbb{C}}^{J} \mid R={ }^{t} R \in \mathbb{C}^{(m, m)}\right\}
$$

is the center of $\mathfrak{g}_{\mathbb{C}}^{J}$.

Lemma 3.1. We have the following.

(1) $[H, E]=2 E, \quad[H, F]=-2 F, \quad[E, F]=H$.

(2) $\left[H, e_{i}\right]=-e_{i}, \quad\left[H, f_{i}\right]=f_{i}, \quad 1 \leq i \leq m$.

(3) $\left[E, e_{i}\right]=f_{i}, \quad\left[E, f_{i}\right]=0, \quad 1 \leq i \leq m$.

(4) $\left[F, e_{i}\right]=0, \quad\left[F, f_{i}\right]=-e_{i}, \quad 1 \leq i \leq m$.

(5) $\left[e_{i}, f_{j}\right]=2 R_{i j}, \quad 1 \leq i, j \leq m$.

Proof. The proof follows immediately from the fact that

$$
\begin{aligned}
& {\left[\left(X_{1},\left(P_{1}, Q_{1}, R_{1}\right)\right),\left(X_{2},\left(P_{2}, Q_{2}, R_{2}\right)\right)\right] } \\
= & \left(\left[X_{1}, X_{2}\right],\left(\left(P_{1}, Q_{1}\right) X_{2}-\left(P_{2}, Q_{2}\right) X_{1}, P_{1}{ }^{t} Q_{2}-P_{2}{ }^{t} Q_{1}+Q_{2}{ }^{t} P_{1}-Q_{1}{ }^{t} P_{2}\right)\right),
\end{aligned}
$$

where $X_{1}, X_{2} \in \mathfrak{s l}_{2}(\mathbb{C}),\left[X_{1}, X_{2}\right]=X_{1} X_{2}-X_{2} X_{1}, \quad P_{i}, Q_{i} \in \mathbb{C}^{(m, 1)}(i=$ $1,2), R_{1}, R_{2} \in \mathbb{C}^{(m, m)}$ with $R_{1}={ }^{t} R_{1}$ and $R_{2}={ }^{t} R_{2}$.

Formally we put

$$
e:=\left(\begin{array}{c}
e_{1} \\
e_{2} \\
\vdots \\
e_{m}
\end{array}\right), \quad f:=\left(\begin{array}{c}
f_{1} \\
f_{2} \\
\vdots \\
f_{m}
\end{array}\right) \text {, }
$$


and

$$
R:=\left(\begin{array}{cccc}
R_{11} & R_{12} & \cdots & R_{1 m} \\
R_{21} & R_{22} & \cdots & R_{2 m} \\
\vdots & \vdots & \ddots & \vdots \\
R_{m 1} & R_{m 2} & \cdots & R_{m m}
\end{array}\right), \quad R_{k l}=R_{l k}, \quad 1 \leq k, l \leq m .
$$

Theorem 3.1. The center $z_{m}\left(\mathfrak{g}_{\mathbb{C}}^{J}\right)$ of the universal enveloping algebra $U\left(\mathfrak{g}_{\mathbb{C}}^{J}\right)$ of $\mathfrak{g}_{\mathbb{C}}^{J}$ is given by

$$
z_{m}\left(\mathfrak{g}_{\mathbb{C}}^{J}\right)=\mathbb{C}\left[\Omega_{m}, R_{k l} \mid 1 \leq k \leq l \leq m\right] .
$$

That is, $\boldsymbol{Z}_{m}\left(\mathfrak{g}_{\mathbb{C}}^{J}\right)$ is a polynomial algebra on $1+\frac{m(m+1)}{2}$ generators $\Omega_{m}, R_{k l}(1 \leq$ $k \leq l \leq m)$. Here

$$
\begin{aligned}
\Omega_{m}:= & \operatorname{det} R\left\{H^{2}-(m+2) H+4 E F\right\} \\
& +\operatorname{det} R\left\{E^{t} e R^{-1} e-{ }^{t} f R^{-1} f F-\left(H-\frac{m+3}{2}\right){ }^{t} f R^{-1} e\right\} \\
& +\operatorname{det} R\left\{\frac{1}{4}{ }^{t} f\left({ }^{t} f R^{-1} e\right) R^{-1} e-\frac{1}{4}\left({ }^{t} e R^{-1} f\right)\left({ }^{t} e R^{-1} e\right)\right\}
\end{aligned}
$$

is a Casimir operator of $\mathcal{U}\left(\mathfrak{g}^{J} \mathbb{C}\right)$ of degree $m+2$.

Proof. Using the method computing the center of the universal enveloping algebra of a certain class of semidirect sum Lie algebras invented by Campoamer-Stursburg and Low [6] (cf. [2], [15]), Conley and Raum [5] proved the above theorem. We refer to [5] for the detail.

Let $\gamma: G^{J} \times\left(\mathbb{H} \times \mathbb{C}^{m}\right) \longrightarrow \mathbb{C}^{\times}$be a scalar cocycle with respect to the action (2.1). This means that $\gamma$ is a smooth function satisfying the cocycle condition

$$
\gamma\left(g_{1} g_{2},(\tau, z)\right)=\gamma\left(g_{1}, g_{2} \cdot(\tau, z)\right) \gamma\left(g_{2},(\tau, z)\right)
$$

for all $g_{1}, g_{2} \in G^{J}$ and $(\tau, z) \in \mathbb{H} \times \mathbb{C}^{m}$. Then we get the map

$$
\widehat{\gamma}(g): G^{J} \longrightarrow C^{\infty}\left(\mathbb{H} \times \mathbb{C}^{m}\right)
$$

defined by

$$
\widehat{\gamma}(g)(\tau, z):=\gamma(g,(\tau, z)), \quad g \in G^{J},(\tau, z) \in \mathbb{H} \times \mathbb{C}^{m} .
$$

Then we obtain the right action $\left.\right|_{\gamma}$ of $G^{J}$ on $C^{\infty}\left(\mathbb{H} \times \mathbb{C}^{m}\right)$ defined by

$$
(g \cdot f)(\tau, z):=\left(\left.f\right|_{\gamma}\left[g^{-1}\right]\right)(\tau, z):=\gamma\left(g^{-1},(\tau, z)\right) f\left(g^{-1} \cdot(\tau, z)\right),
$$

where $g \in G^{J}, f \in C^{\infty}\left(\mathbb{H} \times \mathbb{C}^{m}\right)$ and $(\tau, z) \in \mathbb{H} \times \mathbb{C}^{m}$.

We note that the differential $d \widehat{\gamma}$ of $\widehat{\gamma}$ at the identity is given by

$$
d \widehat{\gamma}(Y)(\tau, z)=\left.\frac{d}{d t}\right|_{t=0} \gamma(\exp (t Y),(\tau, z)) .
$$


Therefore we have the differential right action $\left.\right|_{\gamma}$ of $\mathfrak{g}_{\mathbb{C}}^{J}$ on $C^{\infty}\left(\mathbb{H} \times \mathbb{C}^{m}\right)$ defined by

$$
\begin{aligned}
\left(\left.\phi\right|_{\gamma}[Y]\right)(\tau, z): & =\left.\frac{d}{d t}\right|_{t=0}(\gamma(\exp (t Y),(\tau, z)) \phi(\exp (t Y) \cdot(\tau, z))) \\
& =\gamma(Y,(\tau, z)) \phi(\tau, z)+\left.\frac{d}{d t}\right|_{t=0} \phi(\exp (t Y),(\tau, z))
\end{aligned}
$$

where $Y \in \mathfrak{g}_{\mathbb{C}}^{J}$ and $\phi \in C^{\infty}\left(\mathbb{H} \times \mathbb{C}^{m}\right)$. The action (3.4) extends to $\mathcal{U}\left(\mathfrak{g}_{\mathbb{C}}^{J}\right)$ as usual, and elements of $\mathcal{U}\left(\mathfrak{g}_{\mathbb{C}}^{J}\right)$ of order $r$ act by differential operators of order $\leq r$.

Let $\mathbb{D}_{\gamma}$ be the algebra of all differential operators $D$ on $\mathbb{H} \times \mathbb{C}^{m}$ satisfying the following condition

$$
\left.(D \phi)\right|_{\gamma}[g]=D\left(\left.\phi\right|_{\gamma}[g]\right)
$$

for all $g \in G^{J}$ and for all $\phi \in C^{\infty}\left(\mathbb{H} \times \mathbb{C}^{m}\right)$. Since $G^{J}$ is connected, $\mathbb{D}_{\gamma}$ is the algebra of all differential operators $\mathbb{D}_{\gamma}$ on $\mathbb{H} \times \mathbb{C}^{m}$ commuting with the $\left.\right|_{\gamma}$-action of $\mathfrak{g}_{\mathbb{C}}^{J}$. In particular, the action $\left.\right|_{\gamma}$ maps the center $z_{m}\left(\mathfrak{g}_{\mathbb{C}}^{J}\right)$ of $\mathcal{U}\left(\mathfrak{g}_{\mathbb{C}}^{J}\right)$ into the center $z_{m}\left(\mathbb{D}_{\gamma}\right)$ of $\mathbb{D}_{\gamma}$.

Throughout this section we let $\mathcal{M}$ be a positive definite half-integral symmetric matrix of degree $m$ and let $k \in \mathbb{Z}^{+}$. We let $\gamma_{k, \mathcal{M}}: G^{J} \times\left(\mathbb{H} \times \mathbb{C}^{m}\right) \longrightarrow \mathbb{C}^{\times}$be the canonical automorphic factor for $G^{J}$ on $\mathbb{H} \times \mathbb{C}^{m}$ defined by

$$
\begin{aligned}
& \gamma_{k, \mathcal{M}}((M,(\lambda, \mu ; \kappa)),(\tau, z)): \\
= & (c \tau+d)^{k} e^{2 \pi i \mathcal{M}[z+\lambda \tau+\mu] c(c \tau+d)^{-1}} e^{-2 \pi i \operatorname{tr}\left(\mathcal{M N}\left(\tau \lambda^{\mathrm{t}} \lambda+2 \lambda^{\mathrm{t}} \mathrm{z}+\kappa+\mu^{\mathrm{t}} \lambda\right)\right)},
\end{aligned}
$$

where $(M,(\lambda, \mu ; \kappa)) \in G^{J}$ with $M=\left(\begin{array}{ll}a & b \\ c & d\end{array}\right) \in S L_{2}(\mathbb{R}),(\lambda, \mu ; \kappa) \in H_{\mathbb{R}}^{(m)}$ and $(\tau, z) \in \mathbb{H} \times \mathbb{C}^{m}$.

For brevity we write

$$
\begin{aligned}
\partial_{\tau}:=\frac{\partial}{\partial \tau}=\frac{1}{2}\left(\frac{\partial}{\partial x}-i \frac{\partial}{\partial y}\right), \quad \partial_{\bar{\tau}}:=\frac{\partial}{\partial \bar{\tau}}=\frac{1}{2}\left(\frac{\partial}{\partial x}+i \frac{\partial}{\partial y}\right), \\
\partial_{z_{j}}:=\frac{\partial}{\partial z_{j}}=\frac{1}{2}\left(\frac{\partial}{\partial u_{j}}-i \frac{\partial}{\partial v_{j}}\right), \quad 1 \leq j \leq m, \\
\partial_{\bar{z}_{j}}:=\frac{\partial}{\partial \bar{z}_{j}}=\frac{1}{2}\left(\frac{\partial}{\partial u_{j}}+i \frac{\partial}{\partial v_{j}}\right), \quad 1 \leq j \leq m, \\
\partial_{z}:={ }^{t}\left(\partial_{z_{1}}, \partial_{z_{2}}, \cdots, \partial_{z_{m}}\right), \quad \partial_{\bar{z}}:={ }^{t}\left(\partial_{\bar{z}_{1}}, \partial_{\bar{z}_{2}}, \cdots, \partial_{\bar{z}_{m}}\right) .
\end{aligned}
$$

Lemma 3.2. Let $\mathcal{M}$ and $k$ be as above. We set $\widetilde{\mathcal{M}}:=2 \pi i \mathcal{M}$. Then we have the following:

(3.11) $\left.\right|_{\gamma_{k, \mathfrak{M}}}[(0,(P, Q, R))]=2 \operatorname{Re}\left({ }^{t}(P \tau+Q) \partial_{z}\right)+2{ }^{t} P \widetilde{\mathcal{M}} z+\operatorname{tr}(R \tilde{\mathcal{M}})$.

$$
\begin{aligned}
& \left.\right|_{\gamma_{k, \mathcal{M}}}[E]=2 \operatorname{Re}\left(\partial_{\tau}\right), \\
& \left.\right|_{\gamma_{k, \mathcal{M}}}[F]=-2 \operatorname{Re}\left(\tau\left(\tau \partial_{\tau}+{ }^{t} z \partial_{z}\right)\right)-k \tau-\tilde{\mathcal{M}}[z], \\
& \left.\right|_{\gamma_{k, M}}[H]=2 \operatorname{Re}\left(2 \tau \partial_{\tau}+{ }^{t} z \partial_{z}\right)+k \text {, } \\
& \text { (3.11) } \left.\left.\right|_{\gamma_{k}, \mathcal{M}}(0,(P, Q, R))\right]=2 \operatorname{Re}\left({ }^{t}(P \tau+Q) \partial_{z}\right)+2{ }^{t} P \mathcal{M} z+\operatorname{tr}(R \tilde{\mathcal{M}}) \text {. }
\end{aligned}
$$


Proof. We observe that if $(X,(P, Q, R)) \in \mathfrak{g}_{\mathbb{C}}^{J}$ with $X \in \mathfrak{s l}_{2}(\mathbb{C}), P, Q \in \mathbb{C}^{(m, 1)}$ and $R={ }^{t} R \in \mathbb{C}^{(m, m)}$, then

(3.12) $\exp ((X,(P, Q, R)))=\left(\exp (X),\left((P, Q) g(X), R-(P, Q) h(X)^{t}(-Q, P)\right)\right)$,

where

$$
\exp (t):=\sum_{n=0}^{\infty} \frac{t^{n}}{n !}, \quad g(t):=\frac{e^{t}-1}{t} \quad \text { and } \quad h(t):=\frac{e^{t}-1-t}{t} .
$$

Using the formula (3.12) we easily obtain the formulas (3.8)-(3.11).

\section{Theorem 3.2.}

$$
\left.\right|_{\gamma_{k, \mathcal{M}}}\left[\Omega_{m}\right]=\operatorname{det}(\widetilde{\mathcal{M}})\left\{k(k-m-2)-2 \mathrm{C}^{k, \mathcal{M}}\right\},
$$

where

$$
\begin{aligned}
\mathrm{e}^{k, \mathcal{M}}:= & -8 y^{2} \partial_{\tau} \partial_{\bar{\tau}}+4 i\left(k-\frac{m}{2}\right) y \partial_{\bar{\tau}} \\
& +2 y^{2}\left(\partial_{\bar{\tau}} \widetilde{\mathcal{M}}^{-1}\left[\partial_{z}\right]+\partial_{\tau} \widetilde{\mathcal{M}}^{-1}\left[\partial_{\bar{z}}\right]\right)-8 y \partial_{\tau}{ }^{t} v \partial_{\bar{z}} \\
& -\frac{1}{2} y^{2}\left\{\widetilde{\mathcal{M}}^{-1}\left[\partial_{\bar{z}}\right] \widetilde{\mathcal{M}}^{-1}\left[\partial_{z}\right]-{ }^{t}\left(\partial_{\bar{z}} \widetilde{\mathcal{M}}^{-1} \partial_{z}\right)^{2}\right\}+2 y\left({ }^{t} v \partial_{\bar{z}}\right)^{t} \partial_{z} \widetilde{\mathcal{M}}^{-1} \partial_{u} \\
& -\frac{i}{2}(2 k-m+1) y^{t} \partial_{\bar{z}} \widetilde{\mathcal{M}}^{-1} \partial_{u}+2^{t} v\left({ }^{t} v \partial_{\bar{z}}\right) \partial_{\bar{z}}+i(2 k-m-1)^{t} v \partial_{\bar{z}} .
\end{aligned}
$$

The operator $\mathfrak{C}^{k, \mathcal{M}}$ generates the image of the $\left.\right|_{\gamma_{k, \mathcal{M}}}$-action of the center $\mathcal{Z}_{m}\left(\mathfrak{g}_{\mathbb{C}}^{J}\right)$. In particular, $\mathrm{C}^{k, \mathcal{M}}$ is an element of the center of $\mathbb{D}_{\gamma_{k, \mathcal{M}}}$.

Proof. We write $\widetilde{\mathcal{M}}=\left(\widetilde{\mathcal{M}}_{p q}\right)$. According to (3.11), we have the relation $\left.\right|_{\gamma_{k, \mathfrak{M}}}\left[R_{p q}\right]=$ $\widetilde{\mathcal{M}}_{p q}$ for all $1 \leq p \leq q \leq m$. The proof follows from Theorem 3.1. and Lemma 3.2 .

\section{Invariant differential operators on $\mathbb{H} \times \mathbb{C}^{m}$}

For brevity we put

$$
T_{1, m}:=\mathbb{C} \times \mathbb{C}^{m} .
$$

We define the real linear map $\Phi_{m}: \mathfrak{p}^{J} \longrightarrow T_{1, m}$ by

$$
\Phi_{m}\left(\left(\begin{array}{cc}
x & y \\
y & -x
\end{array}\right),(P, Q, 0)\right)=(x+i y, P+i Q),
$$

where $\left(\left(\begin{array}{cc}x & y \\ y & -x\end{array}\right),(P, Q, 0)\right) \in \mathfrak{p}^{J}$. Obviously $\Phi_{m}$ is a real linear isomorphism of $\mathfrak{p}^{J}$ onto $T_{1, m}$. 
Let $S(m, \mathbb{R})$ denote the additive group consisting of all $m \times m$ real symmetric matrices. We define the group isomorphism $\theta_{m}: K^{J} \longrightarrow U(1) \times S(m, \mathbb{R})$ by

$$
\theta_{m}\left(\left(\begin{array}{cc}
a & -b \\
b & a
\end{array}\right),(0,0 ; \kappa)\right)=(a+i b, \kappa)
$$

where $\left(\left(\begin{array}{cc}a & -b \\ b & a\end{array}\right),(0,0 ; \kappa)\right) \in K^{J}$.

Theorem 4.1. The adjoint representation $A d$ of $K^{J}$ on $\mathfrak{p}^{J}$ is compatible with the natural action of $U(1) \times S(m, \mathbb{R})$ on $T_{1, m}=\mathbb{C} \times \mathbb{C}^{m}$ defined by

$$
(h, \kappa) \cdot(w, \xi):=\left(h^{2} w, h \xi\right), \quad h \in U(1), \kappa \in S(m, \mathbb{R}), w \in \mathbb{C}, \xi \in \mathbb{C}^{m}
$$

through the map $\Phi_{m}$ and $\theta_{m}$. Precisely if $k^{J} \in K^{J}$ and $\alpha \in \mathfrak{p}^{J}$, then we have the following equality

$$
\Phi_{m}\left(A d\left(k^{J}\right) \alpha\right)=\theta_{m}\left(k^{J}\right) \cdot \Phi_{m}(\alpha) .
$$

We recall that we identified $\mathfrak{p}^{J}$ with $\mathbb{C} \times \mathbb{C}^{m}$.

Proof. We refer to [26] for the proof.

The action (4.3) induces the action of $U(1)$ on the polynomial algebra $\operatorname{Pol}_{[m]}:=$ $\operatorname{Pol}\left(T_{1, m}\right)$. We denote by $\operatorname{Pol}_{[m]}^{U(1)}$ the subalgebra of $\operatorname{Pol}_{[m]}$ consisting of $U(1)$ invariants. We let $\mathbb{D}\left(\mathbb{H} \times \mathbb{C}^{m}\right)$ be the algebra of all differential operators invariant under the action (2.1) of $G^{J}$. According to [7], one gets a canonical linear bijection

$$
\Theta_{[m]}: \operatorname{Pol}_{[m]}^{U(1)} \longrightarrow \mathbb{D}\left(\mathbb{H} \times \mathbb{C}^{m}\right)
$$

of $\operatorname{Pol}_{[m]}^{U(1)}$ onto $\mathbb{D}\left(\mathbb{H} \times \mathbb{C}^{m}\right)$. But $\Theta_{[m]}$ is not multiplicative. The map $\Theta_{[m]}$ is described explicitly as follows. Let $\left\{\eta_{\alpha} \mid 1 \leq \alpha \leq 2(m+1)\right\}$ be a basis of $\mathfrak{p}^{J}$. If $P \in \operatorname{Pol}_{[m]}^{U(1)}$, then

$$
\left(\Theta_{[m]}(P) f\right)\left(g K^{J}\right)=\left[P\left(\frac{\partial}{\partial t_{\alpha}}\right) f\left(g \exp \left(\sum_{\alpha=1}^{2(m+1)} t_{\alpha} \eta_{\alpha}\right) K^{J}\right)\right]_{\left(t_{\alpha}\right)=0},
$$

where $g \in G^{J}$ and $f \in C^{\infty}\left(\mathbb{H} \times \mathbb{C}^{m}\right)$.

Theorem 4.2. $\mathrm{Pol}_{[m]}^{U(1)}$ is generated by

$$
\begin{aligned}
& q(w, \xi)=\operatorname{tr}(w \bar{w}), \\
& \alpha_{k p}(w, \xi)=\operatorname{Re}\left(\xi^{t} \bar{\xi}\right)_{k p}, \quad 1 \leq k \leq p \leq m, \\
& \beta_{l q}(w, \xi)=\operatorname{Im}\left(\xi^{t} \bar{\xi}\right)_{l q}, \quad 1 \leq l<q \leq m, \\
& f_{k p}(w, \xi)=\operatorname{Re}\left(\bar{w} \xi^{t} \xi\right)_{k p}, \quad 1 \leq k \leq p \leq m, \\
& g_{k p}(w, \xi)=\operatorname{Im}\left(\bar{w} \xi^{t} \xi\right)_{k p}, \quad 1 \leq k \leq p \leq m,
\end{aligned}
$$


where $w \in \mathbb{C}$ and $\xi \in \mathbb{C}^{m}$.

Proof. We refer to [9] or [26] for the general case.

We let

$$
w=r+i s \in \mathbb{C} \text { and } \xi={ }^{t}\left(\xi_{1}, \cdots, \xi_{m}\right) \in \mathbb{C}^{m} \text { with } \xi_{k}=\zeta_{k}+i \eta_{k}, 1 \leq k \leq m,
$$

where $r, s, \zeta_{1}, \eta_{1}, \cdots, \zeta_{m}, \eta_{m}$ are real. The invariants $q, \alpha_{k p}, \beta_{l q}, f_{k p}$ and $g_{k p}$ are expressed in terms of $r, s, \zeta_{k}, \eta_{l}(1 \leq k, l \leq m)$ as follows:

$$
\begin{aligned}
q(w, \xi) & =r^{2}+s^{2}, \\
\alpha_{k p}(w, \xi) & =\zeta_{k} \zeta_{p}+\eta_{k} \eta_{p}, \quad 1 \leq k \leq p \leq m, \\
\beta_{l q}(w, \xi) & =\zeta_{q} \eta_{l}-\zeta_{l} \eta_{q}, \quad 1 \leq l<q \leq m, \\
f_{k p}(w, \xi) & =r\left(\zeta_{k} \zeta_{p}-\eta_{k} \eta_{p}\right)+s\left(\zeta_{k} \eta_{p}+\eta_{k} \zeta_{p}\right), \quad 1 \leq k \leq p \leq m, \\
g_{k p}(w, \xi) & =r\left(\zeta_{k} \eta_{p}+\eta_{k} \zeta_{p}\right)-s\left(\zeta_{k} \zeta_{p}-\eta_{k} \eta_{p}\right), \quad 1 \leq k \leq p \leq m .
\end{aligned}
$$

Theorem 4.3. The $\frac{m(m+1)}{2}$ relations

$$
f_{k p}^{2}+g_{k p}^{2}=q \alpha_{k k} \alpha_{p p}, \quad 1 \leq k \leq p \leq m
$$

exhaust all the relations among a complete set of generators $q, \alpha_{k p}, \beta_{l q}, f_{k p}$ and $g_{k p}$ of $\operatorname{Pol}_{[m]}^{U(1)}$ with $1 \leq k \leq p \leq m$ and $1 \leq l<q \leq m$.

Theorem 4.4. The action of $U(1)$ on $P_{0} l_{1, m}$ is not multiplicity-free. In fact, if

$$
\operatorname{Pol}_{[m]}=\sum_{\sigma \in \widehat{U(1)}} m_{\sigma} \sigma
$$

then $m_{\sigma}=\infty$.

For the proofs of the above theorems we refer to [26].

We consider the case $m=1$. For a coordinate $(w, \xi)$ in $T_{1,1}$, we write $w=$ $r+i s, \xi=\zeta+i \eta, r, s, \zeta, \eta$ real. The author [21] proved that the algebra $\operatorname{Pol}_{[1]}^{U(1)}$ is generated by

$$
\begin{aligned}
q(w, \xi) & =\frac{1}{4} w \bar{w}=\frac{1}{4}\left(r^{2}+s^{2}\right), \\
\alpha(w, \xi) & =\xi \bar{\xi}=\zeta^{2}+\eta^{2} \\
\phi(w, \xi) & =\frac{1}{2} \operatorname{Re}\left(\xi^{2} \bar{w}\right)=\frac{1}{2} r\left(\zeta^{2}-\eta^{2}\right)+s \zeta \eta \\
\psi(w, \xi) & =\frac{1}{2} \operatorname{Im}\left(\xi^{2} \bar{w}\right)=\frac{1}{2} s\left(\eta^{2}-\zeta^{2}\right)+r \zeta \eta .
\end{aligned}
$$

In [21], using Formula (3.6) the author calculated explicitly the images

$$
D_{1}=\Theta_{[1]}(q), \quad D_{2}=\Theta_{[1]}(\alpha), \quad D_{3}=\Theta_{[1]}(\phi) \quad \text { and } \quad D_{4}=\Theta_{[1]}(\psi)
$$


of $q, \alpha, \phi$ and $\psi$ under the Halgason map $\Theta_{[1]}$. We can show that the algebra $\mathbb{D}(\mathbb{H} \times \mathbb{C})$ is generated by the following differential operators

$$
\begin{gathered}
D_{1}=y^{2}\left(\frac{\partial^{2}}{\partial x^{2}}+\frac{\partial^{2}}{\partial y^{2}}\right)+v^{2}\left(\frac{\partial^{2}}{\partial u^{2}}+\frac{\partial^{2}}{\partial v^{2}}\right) \\
+2 y v\left(\frac{\partial^{2}}{\partial x \partial u}+\frac{\partial^{2}}{\partial y \partial v}\right), \\
D_{2}=y\left(\frac{\partial^{2}}{\partial u^{2}}+\frac{\partial^{2}}{\partial v^{2}}\right), \\
D_{3}=y^{2} \frac{\partial}{\partial y}\left(\frac{\partial^{2}}{\partial u^{2}}-\frac{\partial^{2}}{\partial v^{2}}\right)-2 y^{2} \frac{\partial^{3}}{\partial x \partial u \partial v} \\
-\left(v \frac{\partial}{\partial v}+1\right) D_{2}
\end{gathered}
$$

and

$$
\begin{aligned}
D_{4}=y^{2} & \frac{\partial}{\partial x}\left(\frac{\partial^{2}}{\partial v^{2}}-\frac{\partial^{2}}{\partial u^{2}}\right)-2 y^{2} \frac{\partial^{3}}{\partial y \partial u \partial v} \\
& -v \frac{\partial}{\partial u} D_{2},
\end{aligned}
$$

where $\tau=x+i y$ and $z=u+i v$ with real variables $x, y, u, v$. Moreover, we have

$$
\begin{aligned}
& D_{1} D_{2}-D_{2} D_{1}=2 y^{2} \frac{\partial}{\partial y}\left(\frac{\partial^{2}}{\partial u^{2}}-\frac{\partial^{2}}{\partial v^{2}}\right) \\
&-4 y^{2} \frac{\partial^{3}}{\partial x \partial u \partial v}-2\left(v \frac{\partial}{\partial v} D_{2}+D_{2}\right) .
\end{aligned}
$$

In particular, the algebra $\mathbb{D}(\mathbb{H} \times \mathbb{C})$ is not commutative. We refer to $[1,21]$ for more detail.

Recently Hiroyuki Ochiai [13] (see also [1]) proved the following result.

Theorem 4.5. We have the following relations
(a) $\left[D_{1}, D_{2}\right]=2 D_{3}$
(b) $\left[D_{1}, D_{3}\right]=2 D_{1} D_{2}-2 D_{3}$
(c) $\left[D_{2}, D_{3}\right]=-D_{2}^{2}$
(d) $\left[D_{4}, D_{1}\right]=0$
(e) $\left[D_{4}, D_{2}\right]=0$
(f) $\left[D_{4}, D_{3}\right]=0$ 
(g) $D_{3}^{2}+D_{4}^{2}=D_{2} D_{1} D_{2}$

These seven relations exhaust all the relations among the generators $D_{1}, D_{2}, D_{3}$ and $D_{4}$ of $\mathbb{D}(\mathbb{H} \times \mathbb{C})$.

Remark 4.1. According to Theorem 4.5, we see that $D_{4}$ is a generator of the center of $\mathbb{D}(\mathbb{H} \times \mathbb{C})$. We observe that the Lapalcian

$$
\Delta_{1 ; A, B}=\frac{4}{A} D_{1}+\frac{4}{B} D_{2} \quad(\text { see }(2.5))
$$

of $\left(\mathbb{H} \times \mathbb{C}, d s_{1 ; A, B}^{2}\right)$ does not belong to the center of $\mathbb{D}(\mathbb{H} \times \mathbb{C})$.

\section{Maass-Jacobi Forms due to Yang}

Using $G^{J}$-invariant differential operators on the Siegel-Jacobi space, we introduce a notion of Maass-Jacobi forms.

Definition 5.1. Let

$$
\Gamma_{1, m}:=S L_{2}(\mathbb{Z}) \ltimes H_{\mathbb{Z}}^{(m)}
$$

be the discrete subgroup of $G^{J}$, where

$$
H_{\mathbb{Z}}^{(m)}=\left\{(\lambda, \mu ; \kappa) \in H_{\mathbb{R}}^{(m)} \mid \lambda, \mu, \kappa \text { are integral }\right\} .
$$

A smooth function $f: \mathbb{H} \times \mathbb{C}^{m} \longrightarrow \mathbb{C}$ is called a Maass-Jacobi form on $\mathbb{H} \times \mathbb{C}^{m}$ if $f$ satisfies the following conditions (MJ1)-(MJ3):

(MJ1) $f$ is invariant under $\Gamma_{1, m}$.

(MJ2) $f$ is an eigenfunction of the Laplacian $\Delta_{m ; A, B}$ (cf. Formula (2.5)).

(MJ3) $f$ has a polynomial growth, that is, there exist a constant $C>0$ and a positive integer $N$ such that

$$
|f(x+i y, z)| \leq C|p(y)|^{N} \quad \text { as } y \longrightarrow \infty,
$$

where $p(y)$ is a polynomial in $y$.

Remark 5.1. Let $\mathbb{D}_{*}$ be a commutative subalgebra of $\mathbb{D}\left(\mathbb{H} \times \mathbb{C}^{m}\right)$ containing the Laplacian $\Delta_{m ; A, B}$. We say that a smooth function $f: \mathbb{H} \times \mathbb{C}^{m} \longrightarrow \mathbb{C}$ is a MaassJacobi form with respect to $\mathbb{D}_{*}$ if $f$ satisfies the conditions $(M J 1),(M J 2)_{*}$ and $(M J 3)$ : the condition $(M J 2)_{*}$ is given by

$(M J 2)_{*} f$ is an eigenfunction of any invariant differential operator in $\mathbb{D}_{*}$.

It is natural to propose the following problems.

Problem A : Find all the eigenfunctions of $\Delta_{m ; A, B}$.

Problem B : Construct Maass-Jacobi forms. 
Problem C : Develop the spectral theory of the Laplacian $\Delta_{m ; A, B}$ on a fundamental domain for the Siegel-Jacobi space $\mathbb{H} \times \mathbb{C}^{m}$ with respect to $\Gamma_{1, m}$.

If we find a nice eigenfunction $\phi$ of the Laplacian $\Delta_{m ; A, B}$, we can construct a Maass-Jacobi form $f_{\phi}$ on $\mathbb{H} \times \mathbb{C}^{m}$ in the usual way defined by

$$
f_{\phi}(\tau, z):=\sum_{\gamma \in \Gamma_{1, m}^{\infty} \backslash \Gamma_{1, m}} \phi(\gamma \cdot(\tau, z)),
$$

where

$$
\Gamma_{1, m}^{\infty}=\left\{\left(\left(\begin{array}{ll}
a & b \\
c & d
\end{array}\right),(\lambda, \mu ; \kappa)\right) \in \Gamma_{1, m} \mid c=0\right\}
$$

is a subgroup of $\Gamma_{1, m}$.

We consider the simple case $m=1$ and $A=B=1$. We take a coordinate $(\tau, z) \in \mathbb{H} \times \mathbb{C}$ with $\tau=x+i y, x \in \mathbb{R}, y>0$ and $z=u+i v, u, v$ real. A metric $d s_{1 ; 1,1}^{2}$ on $\mathbb{H} \times \mathbb{C}$ given by

$$
\begin{gathered}
d s_{1 ; 1,1}^{2}=\frac{y+v^{2}}{y^{3}}\left(d x^{2}+d y^{2}\right)+\frac{1}{y}\left(d u^{2}+d v^{2}\right) \\
-\frac{2 v}{y^{2}}(d x d u+d y d v)
\end{gathered}
$$

is a $G^{J}$-invariant Kähler metric on $\mathbb{H} \times \mathbb{C}$. Its Laplacian $\Delta_{1 ; 1,1}$ is given by

$$
\begin{aligned}
\Delta_{1 ; 1,1}= & y^{2}\left(\frac{\partial^{2}}{\partial x^{2}}+\frac{\partial^{2}}{\partial y^{2}}\right) \\
+ & \left(y+v^{2}\right)\left(\frac{\partial^{2}}{\partial u^{2}}+\frac{\partial^{2}}{\partial v^{2}}\right) \\
& +2 y v\left(\frac{\partial^{2}}{\partial x \partial u}+\frac{\partial^{2}}{\partial y \partial v}\right) .
\end{aligned}
$$

We provide some examples of eigenfunctions of $\Delta_{1 ; 1,1}$.

Here

(1) $h(x, y)=y^{\frac{1}{2}} K_{s-\frac{1}{2}}(2 \pi|a| y) e^{2 \pi i a x}(s \in \mathbb{C}, a \neq 0)$ with eigenvalue $s(s-1)$.

$$
K_{s}(z):=\frac{1}{2} \int_{0}^{\infty} \exp \left\{-\frac{z}{2}\left(t+t^{-1}\right)\right\} t^{s-1} d t,
$$

where $\operatorname{Re} z>0$.

(2) $y^{s}, y^{s} x, y^{s} u(s \in \mathbb{C})$ with eigenvalue $s(s-1)$.

(3) $y^{s} v, y^{s} u v, y^{s} x v$ with eigenvalue $s(s+1)$.

(4) $x, y, u, v, x v, u v$ with eigenvalue 0 .

(5) All Maass wave forms. 
We let $f ; \mathbb{H} \times \mathbb{C} \longrightarrow \mathbb{C}$ be a Maass-Jacobi form with $\Delta_{1 ; 1,1} f=\Lambda f$. Then $f$ satisfies the following invariance relations

$$
f(\tau+n, z)=f(\tau, z) \quad \text { for all } n \in \mathbb{Z}
$$

and

$$
f\left(\tau, z+n_{1} \tau+n_{2}\right)=f(\tau, z) \quad \text { for all } n_{1}, n_{2} \in \mathbb{Z} .
$$

Therefore $f$ is a smooth function on $\mathbb{H} \times \mathbb{C}$ which is periodic in $x$ and $u$ with period 1. So $f$ has the following Fourier series

$$
f(\tau, z)=\sum_{n \in \mathbb{Z}} \sum_{r \in \mathbb{Z}} c_{n, r}(y, v) e^{2 \pi i(n x+r u)} .
$$

For two fixed integers $n$ and $r$, for brevity, we set $\varphi(y, v)=c_{n, r}(y, v)$. Then $\varphi$ satisfies the following differential equation

$$
\left[y^{2} \frac{\partial^{2}}{\partial y^{2}}+\left(y+v^{2}\right) \frac{\partial^{2}}{\partial v^{2}}+2 y v \frac{\partial^{2}}{\partial y \partial v}-\left\{(A y+B v)^{2}+B^{2} y+\Lambda\right\}\right] \varphi=0
$$

where $A=2 \pi n$ and $B=2 \pi r$ are constants. We note that the function $\phi(y)=$ $y^{\frac{1}{2}} K_{s-\frac{1}{2}}(2 \pi|n| y)$ satisfies the the differential equation (5.4) with $\Lambda=s(s-1)$. Here $K_{s}(z)$ is the $K$-Bessel function defined by (5.2) (cf. [10], [19]).

\section{Maass-Jacobi forms due to Pitale, Bringmann et al}

We fix a positive integer $m$. Let $\mathcal{M}$ be a symmetric half-integral semi-positive definite matrix of degree $m$. Let $C^{\infty}\left(\mathbb{H} \times \mathbb{C}^{m}\right)$ be the algebra of all $C^{\infty}$-functions on $\mathbb{H} \times \mathbb{C}^{m}$. For any nonnegative integer $k \in \mathbb{Z}$, we define the $\left.\right|_{k, \mathcal{M}}$-slash action of $G^{J}$ on $C^{\infty}\left(\mathbb{H} \times \mathbb{C}^{m}\right)$ as follows: If $f \in C^{\infty}\left(\mathbb{H} \times \mathbb{C}^{m}\right)$, and $(M,(\lambda, \mu ; \kappa)) \in G^{J}$ with $\left(\begin{array}{ll}a & b \\ c & d\end{array}\right) \in S L_{2}(\mathbb{R})$ and $(\lambda, \mu ; \kappa) \in H_{\mathbb{R}}^{(m)}$,

$$
\begin{aligned}
& \left(\left.f\right|_{k, \mathcal{M}}[(M,(\lambda, \mu ; \kappa))]\right)(\tau, z): \\
= & (c \tau+d)^{-k} e^{-2 \pi i \mathcal{M}[z+\lambda \tau+\mu] c(c \tau+d)^{-1}} \\
\times & e^{2 \pi i \operatorname{tr}\left(\mathcal{M}\left(\tau \lambda^{t} \lambda+2 \lambda^{t} z+\kappa+\mu^{t} \lambda\right)\right)} f\left(\frac{a \tau+b}{c \tau+d}, \frac{z+\lambda \tau+\mu}{c \tau+d}\right),
\end{aligned}
$$

where $\tau \in \mathbb{H}$ and $z \in \mathbb{C}^{m}$. We recall the Siegel's notation $\alpha[\beta]={ }^{t} \beta \alpha \beta$ for suitable matrices $\alpha$ and $\beta$. Let $\mathbb{D}_{k, \mathcal{M}}$ be the algebra of all differential operators $D$ on $\mathbb{H} \times \mathbb{C}^{m}$ satisfying the following condition

$$
\left.(D f)\right|_{k, \mathcal{M}}[g]=D\left(\left.f\right|_{k, \mathcal{M}}[g]\right)
$$

for all $f \in C^{\infty}\left(\mathbb{H} \times \mathbb{C}^{m}\right)$ and for all $g \in G^{J}$. We recall the arithmetic subgroup $\Gamma_{1, m}$ of $G^{J}$ defined by

$$
\Gamma_{1, m}:=S L_{2}(\mathbb{Z}) \ltimes H_{\mathbb{Z}}^{(m)} .
$$


Definition 6.1. Let $\mathcal{C}^{k, \mathcal{M}}$ be the Casimir operator defined in Theorem 3.2. A smooth function $\phi: \mathbb{H} \times \mathbb{C}^{m} \longrightarrow \mathbb{C}$ is called a Maass-Jacobi form of weight $k$ and index $\mathcal{M}$ if it satisfies the following conditions:

$\left.\left(\mathrm{MJ} 1^{*}\right) \quad \phi\right|_{k, \mathcal{M}}[\gamma]=\phi$ for all $\gamma \in \Gamma_{1, m}$.

$\left(\mathrm{MJ}^{*}\right) \phi$ is an eigenfunction of the Casimir operator $\mathrm{C}^{k, \mathcal{M}}$.

$\left(\mathrm{MJ}^{*}\right)$ For some $a>0$,

$$
\phi(\tau, z)=O\left(e^{a y} e^{2 \pi i \mathcal{M}[v] / y}\right) \quad \text { as } y \longrightarrow \infty .
$$

Furthermore if $\mathrm{e}^{k, \mathcal{M}} \phi=0$, it is said to be a harmonic Maass-Jacobi form of weight $k$ and index $\mathcal{M}$. We denote by $\mathbb{J}_{k, \mathcal{M}}$ the space of all harmonic Maass-Jacobi forms of weight $k$ and index $\mathcal{M}$.

For the present being we let $\mathcal{M}$ be a positive definite integral even lattice of rank $m$ and $k$ an integer. We identify $\mathcal{M}$ with its Gram matrix with respect to a fixed basis, that is, a positive definite half-integral symmetric matrix of degree $m$. We write $|\mathcal{M}|$ for the determinant of the Gram matrix of $\mathcal{M}$. Throughout this section $n$ will be an integer and $r$ will be in $\mathbb{Z}^{m}$. For $r={ }^{t}\left(r_{1}, \cdots, r_{m}\right) \in \mathbb{Z}^{m}$ and $z={ }^{t}\left(z_{1}, \cdots, z_{m}\right) \in \mathbb{C}^{m}$, we put

$$
\zeta^{r}:=\prod_{j=1}^{m} e^{2 \pi i r_{j} z_{j}},
$$

where $\zeta=\left(\zeta_{1}, \cdots, \zeta_{m}\right)$ with $\zeta_{j}=e^{2 \pi i z_{j}}(1 \leq j \leq m)$. For $a \in \mathbb{C}$, we write $e(a):=e^{2 \pi i a}$. For two vectors $\xi={ }^{t}\left(\xi_{1}, \cdots, \xi_{m}\right)$ and $\eta={ }^{t}\left(\eta_{1}, \cdots, \eta_{m}\right)$ in $\mathbb{C}^{m}$, we let

$$
\langle\xi, \eta\rangle:=\sum_{j=1}^{m} \xi_{j} \eta_{j}
$$

be the standard scalar product.

We set

$$
D=D_{\mathcal{M}}(n, r):=|\mathcal{M}|\left(4 n-\mathcal{M}^{-1}[r]\right) \quad \text { and } \quad h=h_{\mathcal{M}}(r):=|\mathcal{M}| \mathcal{M}^{-1}[r] .
$$

Let $M_{\nu, \mu}(w)$ be the usual $M$-Whittaker function, which is a solution to the following differential equation

$$
\frac{\partial^{2}}{\partial w^{2}} f(w)+\left(-\frac{1}{4}+\frac{\nu}{w}+\frac{\frac{1}{4}-\mu^{2}}{w^{2}}\right) f(w)=0 .
$$

For $s \in \mathbb{C}, \kappa \in \frac{1}{2} \mathbb{Z}$ and $t \in \mathbb{R}^{\times}$, we define the function

$$
\mathcal{M}_{s, \kappa}(t):=|t|^{-\frac{\kappa}{2}} M_{\operatorname{sgn}(t) \frac{\kappa}{2}, s-\frac{1}{2}}(|t|)
$$


and

$$
\phi_{k, \mathcal{M}, s}^{(n, r)}(\tau, z):=\mathcal{M}_{s, k-\frac{m}{2}}\left(\frac{\pi D y}{|\mathcal{M}|}\right) e^{2 \pi i\left(\langle r, z\rangle+\frac{i}{4} \mathcal{M}^{-1}[r] y+n x\right)} .
$$

We define the Poincaré series

$$
P_{k, \mathcal{M}, s}^{(n, r)}(\tau, z):=\sum_{\gamma \in \Gamma_{1, m}^{\infty} \backslash \Gamma_{1, m}}\left(\left.\phi_{s, \mathcal{M}, s}^{(n, r)}\right|_{k, \mathcal{M}}[r]\right)(\tau, z) .
$$

Obviously $P_{k, \mathcal{M}, s}^{(n, r)}$ is holomorphic in $\mathbb{C}^{m}$. It is easily seen that $P_{k, \mathcal{M}, s}^{(n, r)}$ is an eigenfunction of the Casimir operator $\mathfrak{C}^{k, \mathcal{M}}$ with eigenvalue

$$
-2 s(1-s)-\frac{1}{2}\left\{k^{2}-k(m+2)+\frac{1}{4} m(m+4)\right\} .
$$

For $s \in \mathbb{C}, \kappa \in \frac{1}{2} \mathbb{Z}$ and $t \in \mathbb{R}^{\times}$, we set

$$
\mathcal{W}_{s, \kappa}(t):=|t|^{-\frac{\kappa}{2}} W_{\operatorname{sgn}(t) \frac{\kappa}{2}, s-\frac{1}{2}}(|t|),
$$

where $W_{\nu, \mu}$ denotes the usual $W$-Whittaker function which is also a solution to the differential equation (6.4).

For $r \in \mathbb{Z}^{m}$, we define the theta series

$$
\theta_{k, \mathcal{M}}^{(r)}(\tau, z):=\sum_{\lambda \in \mathbb{Z}^{m}} e^{2 \pi i \mathcal{M}[\lambda]} \zeta^{2 \mathcal{M} \lambda}\left\{e^{2 \pi i\langle r, \lambda\rangle} \zeta^{r}+(-1)^{k} e^{-2 \pi i\langle r, \lambda\rangle} \zeta^{r}\right\}
$$

Theorem 6.1(Bringmann-Richter [4] and Conley-Raum [5]). The Poincaré series $P_{s, \mathcal{M}, s}^{(n, r)}(\tau, z)$ has the Fourier expansion

$$
\begin{aligned}
P_{k, \mathcal{M}, s}^{(n, r)}(\tau, z)= & \mathcal{M}_{s, k-\frac{m}{2}}\left(\frac{\pi D y}{|\mathcal{M}|}\right) e\left(\frac{-i D y}{4|\mathcal{M}|}\right) \theta_{k, \mathcal{M}}^{(r)}(\tau, z) e^{2 \pi i n \tau} \\
& +\sum_{n^{\prime} \in \mathbb{Z}, r^{\prime} \in \mathbb{Z}^{m}} c_{y, s}\left(n^{\prime}, r^{\prime}\right) e^{2 \pi i n^{\prime} \tau} \zeta^{r^{\prime}} .
\end{aligned}
$$

Here the coefficients $c_{y, s}\left(n^{\prime}, r^{\prime}\right)$ are

$$
c_{y, s}\left(n^{\prime}, r^{\prime}\right):=b_{y, s}\left(n^{\prime}, r^{\prime}\right)+(-1)^{k} b_{y, s}\left(n^{\prime},-r^{\prime}\right)
$$

with $b_{y, s}$ depending on $D$ and $D^{\prime}=|\mathcal{M}|\left(4 n^{\prime}-\mathcal{M}^{-1}\left[r^{\prime}\right]\right)$ and $b_{y, s}\left(n^{\prime}, r^{\prime}\right)$ is given as follows:

(1) If $D^{\prime}=0$, there is a constant $a_{s}\left(n^{\prime}, r^{\prime}\right)$ such that

$$
b_{y, s}\left(n^{\prime}, r^{\prime}\right)=a_{s}\left(n^{\prime}, r^{\prime}\right) \frac{y^{1+\frac{m}{4}-\frac{k}{2}-s}}{\Gamma\left(s+\frac{k}{2}-\frac{m}{4}\right) \Gamma\left(s-\frac{k}{2}+\frac{m}{4}\right)} .
$$


(2) If $D D^{\prime}>0$,

$$
\begin{aligned}
b_{y, s}\left(n^{\prime}, r^{\prime}\right)= & 2^{1-\frac{m}{2}} \pi i^{-k}|\mathcal{M}|^{-\frac{1}{2}} \frac{\Gamma(2 s)}{\Gamma\left(s-\operatorname{sgn}\left(D^{\prime}\right)\left(\frac{k}{2}-\frac{m}{4}\right)\right)} \\
& \times\left(\frac{D^{\prime}}{D}\right)^{\frac{k}{2}-\frac{m+2}{4}} e\left(-\frac{i D^{\prime} y}{4|\mathcal{M}|}\right) \mathcal{W}_{s, k-\frac{m}{2}}\left(\frac{\pi D^{\prime} y}{|\mathcal{M}|}\right) \\
& \times \sum_{c \in \mathbb{Z}^{+}} c^{-\frac{m+2}{2}} K_{c, \mathcal{M}}\left(n, r, n^{\prime}, r^{\prime}\right) J_{2 s-1}\left(\frac{\pi \sqrt{D D^{\prime}}}{c|\mathcal{M}|}\right),
\end{aligned}
$$

where $\Gamma$ is the usual Gamma function, $J_{s}$ is the usual $J$-Bessel function and $K_{c, \mathcal{M}}\left(n, r, n^{\prime}, r^{\prime}\right)$ is the Kloosterman sum defined by

(6.11) $K_{c, \mathcal{M}}\left(n, r, n^{\prime}, r^{\prime}\right) \quad:=e^{-\pi i c^{-1}\left\langle r, \mathcal{M}^{-1} r^{\prime}\right\rangle}$

$$
\times \sum_{\substack{d \in(\mathbb{Z} / c \mathbb{Z}) \times, \lambda \in \mathbb{Z}^{m} / c \mathbb{Z}^{\prime}}} e^{2 \pi i\left(c^{-1} \bar{d} \mathcal{M}[\lambda]+n^{\prime} d-\left\langle r^{\prime}, \lambda\right\rangle+\bar{d} n+\bar{d}\langle r, \lambda\rangle\right),}
$$

where $\bar{d}$ is an integer inverse of $d$ modulo $c$.

(3) If $D D^{\prime}<0$,

$$
\begin{aligned}
b_{y, s}\left(n^{\prime}, r^{\prime}\right)= & 2^{1-\frac{m}{2}} \pi i^{-k}|\mathcal{M}|^{-\frac{1}{2}} \frac{\Gamma(2 s)}{\Gamma\left(s-\operatorname{sgn}\left(D^{\prime}\right)\left(\frac{k}{2}-\frac{m}{4}\right)\right)} \\
& \times\left(\frac{D^{\prime}}{D}\right)^{\frac{k}{2}-\frac{m+2}{4}} e\left(-\frac{i D^{\prime} y}{4|\mathcal{M}|}\right) \mathcal{W}_{s, k-\frac{m}{2}}\left(\frac{\pi D^{\prime} y}{|\mathcal{M}|}\right) \\
& \times \sum_{c \in \mathbb{Z}^{+}} c^{-\frac{m+2}{2}} K_{c, \mathcal{M}}\left(n, r, n^{\prime}, r^{\prime}\right) I_{2 s-1}\left(\frac{\pi \sqrt{D D^{\prime}}}{c|\mathcal{M}|}\right),
\end{aligned}
$$

where $I_{s}$ is the usual I-Bessel function.

Proof. We refer to [4] for the proof in the case $n=m=1$ and to [5] in the case $n=1, m$ is arbitrary.

Remark 6.1. If $s=\frac{k}{2}-\frac{m}{4}$ (resp. $s=1+\frac{m}{4}-\frac{k}{2}$ ), then the Poincaré series $P_{k, \mathcal{M}, s}^{(n, r)}(\tau, z)$ converges for $k>m+2$ (resp. $\left.k<0\right)$. In both cases Poincaré series $P_{k, \mathcal{M}, s}^{(n, r)}(\tau, z)$ is a harmonic Maass-Jacobi form of weight $k$ and index $\mathcal{M}$ which is holomorphic in $\mathbb{C}^{m}$.

Remark 6.2. The Fourier coefficients $c_{y, s}^{(n, r)}=c_{k, \mathcal{M}, s}^{(n, r)}$ of the Poincaré series $P_{k, \mathcal{M}, s}^{(n, r)}(\tau, z)$ satisfy the the so-called Zagier-type duality with dual weights $k$ and $m+2-k$. More precisely, if $D<0$ and $D^{\prime}<0$, there is a constant $h_{k, s}$ depending only on $k$ and $s$ such that

$$
c_{k, \mathcal{M}, s}^{(n, r)}\left(n^{\prime}, r^{\prime}\right)=h_{k, s} c_{m+2-k, \mathcal{M}, s}^{\left(n^{\prime}, r^{\prime}\right)}(n, r)
$$


while if $D<0$ and $D^{\prime}>0$, there is a constant $\hat{h}_{k, s}$ depending only on $k$ and $s$ such that

$$
c_{k, \mathcal{M}, s}^{(n, r)}\left(n^{\prime}, r^{\prime}\right)=\hat{h}_{k, s} c_{m+2-k, \mathcal{M}, s}^{\left(n^{\prime}, r^{\prime}\right)}(n, r) .
$$

\section{Skew-Holomorphic Jacobi Forms}

We define the skew-slash action of $G^{J}$ on $C^{\infty}\left(\mathbb{H} \times \mathbb{C}^{m}\right)$ as follows: If $f \in$ $C^{\infty}\left(\mathbb{H} \times \mathbb{C}^{m}\right)$, and $(M,(\lambda, \mu ; \kappa)) \in G^{J}$ with $\left(\begin{array}{ll}a & b \\ c & d\end{array}\right) \in S L_{2}(\mathbb{R})$ and $(\lambda, \mu ; \kappa) \in H_{\mathbb{R}}^{(m)}$,

$$
\begin{aligned}
& \left(\left.f\right|_{k, \mathcal{M}} ^{s k}[(M,(\lambda, \mu ; \kappa))]\right)(\tau, z): \\
= & (c \bar{\tau}+d)^{1-k}|c \tau+d|^{-1} e^{-2 \pi i \mathcal{M N}[z+\lambda \tau+\mu] c(c \tau+d)^{-1}} \\
& \times e^{2 \pi i \operatorname{tr}\left(\mathcal{M}\left(\tau \lambda^{\mathrm{t}} \lambda+2 \lambda^{\mathrm{t}} \mathbf{z}+\kappa+\mu^{\mathrm{t}} \lambda\right)\right)} f\left(\frac{a \tau+b}{c \tau+d}, \frac{z+\lambda \tau+\mu}{c \tau+d}\right),
\end{aligned}
$$

where $\tau \in \mathbb{H}$ and $z \in \mathbb{C}^{m}$.

Definition 7.1. A smooth $f: \mathbb{H} \times \mathbb{C}^{m} \longrightarrow \mathbb{C}$ is said to be a skew-holomorphic Jacobi form of weight $k$ and index $\mathcal{M}$ if it is real analytic in $\tau$ and is holomorphic in $z \in \mathbb{C}^{m}$ and satisfies the following conditions:

(SK1) $\left.f\right|_{k, \mathcal{M}} ^{s k}[\gamma]=f$ for all $\gamma \in \Gamma^{J}$.

(SK2) The Fourier expansion of $f$ is of the form

$$
f(\tau, z)=\sum_{\substack{n \in \mathbb{Z}, r \in \mathbb{Z}^{m} \\ D \gg-\infty}} c(n, r) e^{\pi D y /|\mathcal{M}|} e^{2 \pi i n \tau} \zeta^{r} .
$$

We denote by $\mathbb{J}_{k, \mathcal{M}}^{s k}$ the space of all skew-holomorphic Jacobi forms of weight $k$ and index $\mathcal{M}$.

Remark 7.1. The notion of skew-holomorphic Jacobi forms was introduced by N.-P. Skoruppa [18].

Let

$$
e_{n, r, \mathcal{M}}(\tau, z):=e^{2 \pi i(n \tau+\langle r, z\rangle)} e^{\pi D y /|\mathcal{M}|} .
$$

We define the Poincaré series

$$
P_{k, \mathcal{M}}^{(n, r), s k}(\tau, z):=\sum_{\gamma \in \Gamma_{1, m}^{\infty} \backslash \Gamma_{1, m}}\left(\left.e_{n, r, \mathcal{M}}\right|_{k, \mathcal{M}} ^{s k}[\gamma]\right)(\tau, z) .
$$

Theorem 7.1. The Poincaré series $P_{k, \mathcal{M}}^{(n, r), s k}(\tau, z)$ defined in (7.2) is a cuspidal skew-holomorphic Jacobi form of weight $k$ and index $\mathcal{M}$. And it has the Fourier 
expansion

$$
\begin{aligned}
P_{k, \mathcal{M}}^{(n, r), s k}(\tau, z)= & e^{\pi D y /|\mathcal{M}|} \theta_{k-1, \mathcal{M}}^{(r)}(\tau, z) e^{2 \pi i n \tau} \\
& +\sum_{\substack{n^{\prime} \in \mathbb{Z}, r^{\prime} \in \mathbb{Z}^{m} \\
D^{\prime}>0}} c\left(n^{\prime}, r^{\prime}\right) e^{\pi D^{\prime} y /|\mathcal{M}|} e^{2 \pi i n^{\prime} \tau} \zeta^{r^{\prime}},
\end{aligned}
$$

where $\theta_{k, \mathcal{M}}^{(r)}(\tau, z)$ is defined in Formula (6.9) and the coefficients $c\left(n^{\prime}, r^{\prime}\right)$ are

$$
c\left(n^{\prime}, r^{\prime}\right)=b\left(n^{\prime}, r^{\prime}\right)+(-1)^{k} b\left(n^{\prime},-r^{\prime}\right) .
$$

Here

$$
\begin{aligned}
b\left(n^{\prime}, r^{\prime}\right):= & 2^{1-\frac{m}{2}} \pi i^{1-k}\left(\frac{D^{\prime}}{D}\right)^{\frac{k}{2}-\frac{m+2}{4}} \\
& \times \sum_{c \in \mathbb{Z}^{+}} c^{-\frac{m+2}{2}} K_{c, \mathcal{M}}\left(n, r, n^{\prime},-r^{\prime}\right) J_{k-\frac{m+2}{2}}\left(\frac{\pi \sqrt{D D^{\prime}}}{c|\mathcal{M}|}\right) .
\end{aligned}
$$

Proof. The proof is analogous to that of Theorem 6.1.

We define the following lowering operator

$$
\begin{aligned}
D_{-}^{(\mathcal{M})} & =\left(\frac{\tau-\bar{\tau}}{2 i}\right)\left\{-(\tau-\bar{\tau}) \partial_{\bar{\tau}}-{ }^{t}(z-\bar{z}) \partial_{\bar{z}}+\frac{\tau-\bar{\tau}}{8 \pi i} \mathcal{M}^{-1}\left[\partial_{\bar{z}}\right]\right\} \\
& =-2 i y\left(y \partial_{\bar{\tau}}+{ }^{t} v \partial_{\bar{z}}-\frac{y}{8 \pi i} \mathcal{M}^{-1}\left[\partial_{\bar{z}}\right]\right) .
\end{aligned}
$$

We note that $D_{-}^{(\mathcal{M})}$ satisfies the following relation

$$
\left.\left(D_{-}^{(\mathcal{M})} \phi\right)\right|_{k-2, \mathcal{M}}[\gamma]=D_{-}^{(\mathcal{M})}\left(\left.\phi\right|_{k, \mathcal{M}}[\gamma]\right)
$$

for all $\phi \in C^{\infty}\left(\mathbb{H} \times \mathbb{C}^{m}\right)$ and for all $\gamma \in \Gamma_{1, m}$.

Now we define the differential operator

$$
\xi_{k, \mathcal{M}}:=\left(\frac{\tau-\bar{\tau}}{2 i}\right)^{k-\frac{5}{2}} D_{-}^{(\mathcal{M})}=y^{k-\frac{5}{2}} D_{-}^{(\mathcal{M})}
$$

It is easily seen that if $f$ is a harmonic Maass-Jacobi form of weight $k$ and index $\mathcal{M}$ which is holomorphic in $\mathbb{C}^{m}$, then the image $\xi_{k, \mathcal{M}} f$ of $f$ under $\xi_{k, \mathcal{M}}$ is a skewholomorphic Jacobi form of weight $3-k$ and index $\mathcal{M}$.

Theorem 7.2. The Poincaré series $P_{k, \mathcal{M}}^{(n, r), s k}(\tau, z)$ span the space $\mathbb{J}_{k, \mathcal{M}}^{\text {sk,cusp }}$ of all cuspidal skew-holomorphic Jacobi forms of weight $k$ and index $\mathcal{M}$.

Proof. The proof can be found in [18]. 
Now we consider the special case $s=\frac{k}{2}-\frac{m}{4}$ and $s=1+\frac{m}{4}-\frac{k}{2}$.

Proposition 7.1. The Poincaré series $P_{k, \mathcal{M}, \frac{k}{2}-\frac{m}{4}}^{(n, r)}$ with $k>2+m$ is meromorphic. If $k<0$,

$$
\xi_{k, \mathcal{M}}\left(P_{k, \mathcal{M}, 1+\frac{m}{4}-\frac{k}{2}}^{(n, r)}\right)=c_{k, \mathcal{M}} P_{3-k, \mathcal{M}}^{(n, r), s k},
$$

where $c_{k, \mathcal{M}}$ is a constant depending on $k$ and $\mathcal{M}$.

Proof. We refer to [5], p. 18 for the proof.

Proposition 7.2. Let $\mathbb{J}_{k, \mathcal{M}}^{c u s p}$ be the space of all cuspidal harmonic Maass-Jacobi forms of weight $k$ and index $\mathcal{M}$ which are holomorphic in $\mathbb{C}^{m}$. Then we have the relation

$$
\xi_{k, \mathcal{M}}\left(\mathbb{J}_{k, \mathcal{M}}^{c u s p, *}\right)=\mathbb{J}_{k, \mathcal{M}}^{s k, \text { cusp }} .
$$

Proof. We refer to [5], p. 18 for the proof.

\section{Covariant differential operators on $\mathbb{H} \times \mathbb{C}^{m}$}

Let $G$ be a real Lie group, $H$ a closed subgroup and $V$ a finite dimensional complex vector space. For an element $x \in G$ we denote the $\operatorname{coset} x H$ by $\bar{x}$. A 1cocycle of $G$ on $G / H$ with values in $V$ is a smooth function $\alpha: G \times G / H \longrightarrow G L(V)$ satisfying the following condition

$$
\alpha\left(g_{1} g_{2}, \bar{x}\right)=\alpha\left(g_{2}, \bar{x}\right) \alpha\left(g_{1}, g_{2} \bar{x}\right)
$$

for all $g_{1}, g_{2}, x \in G$. The associated right action of $G$ on $C^{\infty}(G / H) \otimes V$ is

$$
\left.f\right|_{\alpha}[g](\bar{x}):=\alpha(g, \bar{x}) f(g \bar{x}), \quad g, x \in G
$$

and the associated representation of $H$ on $V$ is

$$
\pi_{\alpha}(h):=\alpha(h, \bar{x}),
$$

where $h \in H$ and $e$ is the identity element of $G$.

Definition 8.1. Let $V$ and $V^{\prime}$ be two finite dimensional complex vector spaces. Let $\alpha$ and $\alpha^{\prime}$ be two 1-cocycles of $G$ on $G / H$ with values in $V$ and $V^{\prime}$ respectively. A differential operator $D: C^{\infty}(G / H) \otimes V \longrightarrow C^{\infty}(G / H) \otimes V^{\prime}$ is covariant from $\left.\right|_{\alpha}$ to $\left.\right|_{\alpha^{\prime}}$ if for all $g \in G$ and $f \in C^{\infty}(G / H) \otimes V$, we have

$$
D\left(\left.f\right|_{\alpha}[g]\right)=\left.(D f)\right|_{\alpha^{\prime}}[g] .
$$

Let $\mathbb{D}_{\alpha, \alpha^{\prime}}(G / H)$ be the space of all covariant differential operators from $\left.\right|_{\alpha}$ to $\left.\right|_{\alpha^{\prime}}$ and $\mathbb{D}_{\alpha, \alpha^{\prime}}^{q}(G / H)$ be the space of those of order $\leq q$. When $\alpha=\alpha^{\prime}$, we refer to such operators as $\left.\right|_{\alpha}$-invariant, and we write simply $\mathbb{D}_{\alpha}(G / H)$ and $\mathbb{D}_{\alpha}^{q}(G / H)$ 
We consider our case

$$
G^{J}=S L_{2}(\mathbb{R}) \ltimes H_{\mathbb{R}}^{(m)} \quad \text { and } \quad K^{J}=S O(2) \ltimes S(m, \mathbb{R}) .
$$

We observe that $K^{J}$ is an abelian closed subgroup of $G^{J}$. We define the linear map $\xi: \mathfrak{g}_{\mathbb{C}}^{J} \longrightarrow \mathfrak{g}_{\mathbb{C}}^{J}$ by $\xi(X)=\widehat{X}$ with $X \in \mathfrak{g}_{\mathbb{C}}^{J}$, where

$$
\begin{aligned}
\widehat{H}: & =i(F-E), \quad \widehat{E}:=\frac{1}{2}\{H+i(E+F)\}, \quad \widehat{F}:=\frac{1}{2}\{H-i(E+F)\}, \\
\widehat{R}_{k l}: & =\frac{1}{2} R_{k l}, \quad \widehat{e}_{j}:=\frac{1}{2}\left(e_{j}-i f_{j}\right), \quad \widehat{f}_{j}:=\frac{1}{2}\left(e_{j}+i f_{j}\right) .
\end{aligned}
$$

It is easy to see that there is a unique $K^{J}$-splitting

$$
\mathfrak{g}_{\mathbb{C}}^{J}=\mathfrak{k}_{*}^{J} \oplus \mathfrak{p}_{*}^{J},
$$

where

$$
\mathfrak{k}_{*}^{J}=\operatorname{span}\left\{\widehat{H}, \widehat{R}_{k l} \mid 1 \leq k \leq l \leq m\right\}
$$

and

$$
\mathfrak{p}_{*}^{J}=\operatorname{span}\left\{\widehat{E}, \widehat{F}, \widehat{e}_{j}, \widehat{f}_{j} \mid 1 \leq j \leq m\right\} .
$$

We note that $\xi$ is an automorphism of Lie algebras and so the given basis of $\mathfrak{p}_{*}^{J}$ is a $K^{J}$-eigenbasis : the $\widehat{H}$ =weights of $\widehat{E}, \widehat{F}, \widehat{e}_{j}$ and $\widehat{f}_{j}$ are $1,-2,-1$ and 1 respectively. We take the scalar valued 1 -cocycle $\alpha:=\gamma_{k, \mathcal{M}}$ defined by (3.7). We set $\mathcal{M}=\left(\mathcal{M}_{k l}\right)$. We let $\pi_{k, \mathcal{M}}: K^{J} \longrightarrow G L_{1}(\mathbb{C})$ be the one-dimensional representation of $K^{J}$ defined by

$$
\pi_{k, \mathcal{M}}(h):=\gamma_{k, \mathcal{M}}(h, \bar{e})^{-1},
$$

where $h \in K^{J}$ and $\bar{e}=(i, 0)=e K^{J}$ with the identity element $e$ in $G^{J}$. We remark that $\xi$ maps the Casimir operator $\Omega_{m}$ to $\left(\frac{i}{2}\right)^{m} \Omega_{m}$.

Definition 8.2. Let $k \in \mathbb{Z}$ and $\mathcal{M} \in S(m, \mathbb{C})$. We define the raising operators $X_{+}, Y_{+}$and the lowering operators $X_{-}$and $Y_{-}$:

$$
\begin{aligned}
& X_{+}^{k, \mathcal{M}}:=2 i\left(\partial_{\tau}+y^{-1} t v \partial_{z}+y^{-2} \widetilde{\mathcal{M}}[v]\right), \quad X_{-}^{k, \mathcal{M}}:=-2 i y\left(y \partial_{\bar{\tau}}+{ }^{t} v \partial_{\bar{z}}\right), \\
& Y_{+}^{k, \mathcal{M}}:=i \partial_{z}+2 i y^{-1} \widetilde{\mathcal{M}} v, \quad Y_{-}^{k, \mathcal{M}}:=-i y \partial_{\tilde{z}}, \quad \tilde{\mathcal{M}}:=2 \pi i \mathcal{M} .
\end{aligned}
$$

We write $Y_{ \pm, j}^{k, \mathcal{M}}$ for the $j$-th entry of $Y_{ \pm}^{k, \mathcal{M}}(1 \leq j \leq m)$.

For brevity, we write

$$
\mathbb{D}\left(k, \mathcal{M} ; k^{\prime}, \mathcal{M}^{\prime}\right):=\mathbb{D}_{\gamma_{k, \mathfrak{M}}, \gamma_{k^{\prime}, \mathcal{M}^{\prime}}}\left(G^{J} / K^{J}\right)
$$

and

$$
\mathbb{D}^{q}\left(k, \mathcal{M} ; k^{\prime}, \mathcal{M}^{\prime}\right):=\mathbb{D}_{\gamma_{k, \mathcal{M}}, \gamma_{k^{\prime}, \mathcal{M}^{\prime}}}^{q}\left(G^{J} / K^{J}\right),
$$


where $k, k^{\prime} \in \mathbb{Z}, \mathcal{M}, \mathcal{M}^{\prime} \in S(m, \mathbb{C}), q \in \mathbb{Z} \cup\{0\}$ and $G^{J} / K^{J}=\mathbb{H} \times \mathbb{C}^{m}$. We also write

$$
\mathbb{D}_{k, \mathcal{M}}:=\mathbb{D}(k, \mathcal{M} ; k, \mathcal{M}) \quad \text { and } \quad \text { and } \quad \mathbb{D}_{k, \mathcal{M}}^{q}:=\mathbb{D}^{q}(k, \mathcal{M} ; k, \mathcal{M}) .
$$

Conley and Raum [5] obtained the following three results.

Proposition 8.1. (1) The spaces $\mathbb{D}^{1}(k, \mathcal{M} ; k \pm 2, \mathcal{M})$ are one-dimensional. In fact $\mathbb{D}^{1}(k, \mathcal{M} ; k \pm 2, \mathcal{M})=\mathbb{C} X_{ \pm}^{k, \mathcal{M}}$.

(2) $\mathbb{D}^{1}(k, \mathcal{M} ; k \pm 1, \mathcal{M})=\operatorname{Span}\left\{Y_{ \pm, j}^{k, \mathcal{M}} \mid 1 \leq j \leq m\right\}$ are $m$-dimensional.

(3) $\mathbb{D}_{k, \mathcal{M}}^{0}=\mathbb{D}_{k, \mathcal{M}}^{1}=\mathbb{C}$.

(4) All other $\mathbb{D}^{1}\left(k, \mathcal{M} ; k^{\prime}, \mathcal{M}^{\prime}\right)$ are zero.

(5) We have the following commutation relations

$$
\begin{gathered}
{\left[X_{-}, X_{+}\right]=-k, \quad\left[Y_{-, j}, Y_{+, j^{\prime}}\right]=i \tilde{\mathcal{M}}_{j j^{\prime}}, \quad\left[X_{-}, Y_{+}\right]=-Y_{-},} \\
{\left[Y_{-}, X_{+}\right]=Y_{+}, \quad\left[X_{+}, Y_{+}\right]=\left[X_{-}, Y_{-}\right]=0 .}
\end{gathered}
$$

Proposition 8.2. Any covariant differential operator of order $q$ may be expressed as a linear combination of products up to $q$ raising and lowering operators. There is a unique such expression in which the raising operators are all to the left of the lowering operators. The expression of this form for the Casimir operator $\mathrm{C}^{k, \mathrm{M}}$ is

$$
\begin{aligned}
\mathrm{e}^{k, \mathcal{M}}= & -2 X_{+} X_{-}+i\left(X_{+} \tilde{\mathcal{M}}^{-1}\left[Y_{-}\right]-\tilde{\mathcal{M}}^{-1}\left[Y_{+}\right] X_{-}\right) \\
& -\frac{1}{2}\left\{\widetilde{\mathcal{M}}^{-1}\left[Y_{+}\right] \widetilde{\mathcal{M}}^{-1}\left[Y_{-}\right]-{ }^{t} Y_{+}\left({ }^{t} Y_{+} \widetilde{\mathcal{M}}^{-1} Y_{-}\right) \widetilde{\mathcal{M}}^{-1} Y_{-}\right\} \\
& -\frac{i}{2}(2 k-m-3){ }^{t} Y_{+} \widetilde{\mathcal{M}}^{-1} Y_{-} .
\end{aligned}
$$

Proposition 8.3. The algebra $\mathbb{D}_{k, \mathcal{M}}$ is generated by $\mathbb{D}_{k, \mathcal{M}}^{3}$. Bases for $\mathbb{D}_{k, \mathcal{M}}^{2}$ and $\mathbb{D}_{k, \mathfrak{M}}^{3}$ are given by

$$
\begin{aligned}
& \mathbb{D}_{k, \mathcal{M}}^{2}=\operatorname{Span}\left\{1, X_{+} X_{-}, Y_{+, i} Y_{-, j} \mid 1 \leq i, j \leq m\right\}, \\
& \mathbb{D}_{k, \mathcal{M}}^{3}=\operatorname{Span}\left\{X_{+} Y_{-, i} Y_{-, j}, Y_{+, i} Y_{+, j} X_{-} \mid 1 \leq i \leq j \leq m\right\} \oplus \mathbb{D}_{k, \mathcal{M}}^{2} .
\end{aligned}
$$

Therefore we have

$$
\operatorname{dim}_{\mathbb{C}} \mathbb{D}_{k, \mathcal{M}}^{2}=m^{2}+2 \quad \text { and } \quad \operatorname{dim}_{\mathbb{C}} \mathbb{D}_{k, \mathcal{M}}^{3}=2 m^{2}+m+2 .
$$




\section{Final remarks}

In this final section we briefly remark the general case $n>1$ and $m>1$.

We let

$$
\mathbb{H}_{n}=\left\{\Omega \in \mathbb{C}^{(n, n)} \mid \Omega={ }^{t} \Omega, \quad \operatorname{Im} \Omega>0\right\}
$$

be the Siegel upper half plane of degree $n$ and let

$$
S p(n, \mathbb{R})=\left\{M \in \mathbb{R}^{(2 n, 2 n)} \mid{ }^{t} M J_{n} M=J_{n}\right\}
$$

be the symplectic group of degree $n$, where

$$
J_{n}=\left(\begin{array}{cc}
0 & I_{n} \\
-I_{n} & 0
\end{array}\right)
$$

$S p(n, \mathbb{R})$ acts on $\mathbb{H}_{n}$ transitively by

$$
M \cdot \Omega=(A \Omega+B)(C \Omega+D)^{-1},
$$

where $M=\left(\begin{array}{ll}A & B \\ C & D\end{array}\right) \in S p(n, \mathbb{R})$ and $\Omega \in \mathbb{H}_{n}$.

For brevity, we write $G_{n}=S p(n, \mathbb{R})$. The isotropy subgroup $K_{n}$ at $i I_{n}$ for the action (9.1) is a maximal compact subgroup given by

$$
K_{n}=\left\{\left(\begin{array}{cc}
A & -B \\
B & A
\end{array}\right) \mid A^{t} A+B^{t} B=I_{n}, A^{t} B=B^{t} A, A, B \in \mathbb{R}^{(n, n)}\right\} .
$$

Let $\mathfrak{k}_{n}$ be the Lie algebra of $K_{n}$. Then the Lie algebra $\mathfrak{g}_{n}$ of $G_{n}$ has a Cartan decomposition $\mathfrak{g}_{n}=\mathfrak{k}_{n} \oplus \mathfrak{p}_{n}$, where

$$
\begin{gathered}
\mathfrak{g}_{n}=\left\{\left(\begin{array}{cc}
X_{1} & X_{2} \\
X_{3} & -{ }^{t} X_{1}
\end{array}\right) \mid X_{1}, X_{2}, X_{3} \in \mathbb{R}^{(n, n)}, X_{2}={ }^{t} X_{2}, X_{3}={ }^{t} X_{3}\right\}, \\
\mathfrak{k}_{n}=\left\{\left(\begin{array}{cc}
X & -Y \\
Y & X
\end{array}\right) \in \mathbb{R}^{(2 n, 2 n)} \mid{ }^{t} X+X=0, Y={ }^{t} Y\right\}, \\
\mathfrak{p}_{n}=\left\{\left(\begin{array}{cc}
X & Y \\
Y & -X
\end{array}\right) \mid X={ }^{t} X, Y={ }^{t} Y, X, Y \in \mathbb{R}^{(n, n)}\right\} .
\end{gathered}
$$

The subspace $\mathfrak{p}_{n}$ of $\mathfrak{g}_{n}$ may be regarded as the tangent space of $\mathbb{H}_{n}$ at $i I_{n}$.

We consider the Heisenberg group

$$
H_{\mathbb{R}}^{(n, m)}=\left\{(\lambda, \mu ; \kappa) \mid \lambda, \mu \in \mathbb{R}^{(m, n)}, \kappa \in \mathbb{R}^{(m, m)}, \kappa+\mu^{t} \lambda \text { symmetric }\right\}
$$

endowed with the following multiplication law

$$
(\lambda, \mu ; \kappa) \circ\left(\lambda^{\prime}, \mu^{\prime} ; \kappa^{\prime}\right)=\left(\lambda+\lambda^{\prime}, \mu+\mu^{\prime} ; \kappa+\kappa^{\prime}+\lambda^{t} \mu^{\prime}-\mu^{t} \lambda^{\prime}\right)
$$


with $(\lambda, \mu ; \kappa),\left(\lambda^{\prime}, \mu^{\prime} ; \kappa^{\prime}\right) \in H_{\mathbb{R}}^{(n, m)}$. We define the semidirect product of $S p(n, \mathbb{R})$ and $H_{\mathbb{R}}^{(n, m)}$

$$
G_{n, m}^{J}=S p(n, \mathbb{R}) \ltimes H_{\mathbb{R}}^{(n, m)}
$$

endowed with the following multiplication law

$$
(M,(\lambda, \mu ; \kappa)) \cdot\left(M^{\prime},\left(\lambda^{\prime}, \mu^{\prime} ; \kappa^{\prime}\right)\right)=\left(M M^{\prime},\left(\tilde{\lambda}+\lambda^{\prime}, \tilde{\mu}+\mu^{\prime} ; \kappa+\kappa^{\prime}+\tilde{\lambda}^{t} \mu^{\prime}-\tilde{\mu}^{t} \lambda^{\prime}\right)\right)
$$

with $M, M^{\prime} \in S p(n, \mathbb{R}),(\lambda, \mu ; \kappa),\left(\lambda^{\prime}, \mu^{\prime} ; \kappa^{\prime}\right) \in H_{\mathbb{R}}^{(n, m)}$ and $(\tilde{\lambda}, \tilde{\mu})=(\lambda, \mu) M^{\prime}$. Then $G_{n, m}^{J}$ acts on $\mathbb{H}_{n} \times \mathbb{C}^{(m, n)}$ transitively by

$$
(M,(\lambda, \mu ; \kappa)) \cdot(\Omega, Z)=\left(M \cdot \Omega,(Z+\lambda \Omega+\mu)(C \Omega+D)^{-1}\right),
$$

where $M=\left(\begin{array}{ll}A & B \\ C & D\end{array}\right) \in S p(n, \mathbb{R}),(\lambda, \mu ; \kappa) \in H_{\mathbb{R}}^{(n, m)}$ and $(\Omega, Z) \in \mathbb{H}_{n} \times \mathbb{C}^{(m, n)}$.

The stabilizer $K_{n, m}^{J}$ of $G_{n, m}^{J}$ at $\left(i I_{n}, 0\right)$ for the action (9.2) is given by

$$
K_{n, m}^{J}=\left\{(k,(0,0 ; \kappa)) \mid k \in K_{n}, \kappa={ }^{t} \kappa \in \mathbb{R}^{(m, m)}\right\} .
$$

Therefore $\mathbb{H}_{n} \times \mathbb{C}^{(m, n)} \cong G_{n, m}^{J} / K_{n, m}^{J}$ is a homogeneous space of non-reductive type. The Lie algebra $\mathfrak{g}_{n, m}^{J}$ of $G_{n, m}^{J}$ has a decomposition

$$
\mathfrak{g}_{n, m}^{J}=\mathfrak{k}_{n, m}^{J}+\mathfrak{p}_{n, m}^{J}
$$

where

$$
\begin{gathered}
\mathfrak{g}_{n, m}^{J}=\left\{(Z,(P, Q, R)) \mid Z \in \mathfrak{g}_{n}, P, Q \in \mathbb{R}^{(m, n)}, R={ }^{t} R \in \mathbb{R}^{(m, m)}\right\}, \\
\mathfrak{k}_{n, m}^{J}=\left\{(X,(0,0, R)) \mid X \in \mathfrak{k}_{n}, R={ }^{t} R \in \mathbb{R}^{(m, m)}\right\}, \\
\mathfrak{p}_{n, m}^{J}=\left\{(Y,(P, Q, 0)) \mid Y \in \mathfrak{p}_{n}, P, Q \in \mathbb{R}^{(m, n)}\right\} .
\end{gathered}
$$

Thus the tangent space of the homogeneous space $\mathbb{H}_{n, m}$ at $\left(i I_{n}, 0\right)$ is identified with $\mathfrak{p}_{n, m}^{J}$. We note that the Jacobi group $G_{n, m}^{J}$ is not a reductive Lie group and that the homogeneous space $\mathbb{H}_{n} \times \mathbb{C}^{(m, n)}$ is not a symmetric space. From now on, for brevity we write $\mathbb{H}_{n, m}=\mathbb{H}_{n} \times \mathbb{C}^{(m, n)}$, called the Siegel-Jacobi space of degree $n$ and index $m$.

For a coordinate $(\Omega, Z) \in \mathbb{H}_{n, m}$ with $\Omega=\left(\omega_{\mu \nu}\right) \in \mathbb{H}_{n}$ and $Z=\left(z_{k l}\right) \in \mathbb{C}^{(m, n)}$, we put

$$
\begin{aligned}
\Omega & =X+i Y, \quad X=\left(x_{\mu \nu}\right), \quad Y=\left(y_{\mu \nu}\right) \text { real, } \\
Z & =U+i V, \quad U=\left(u_{k l}\right), \quad V=\left(v_{k l}\right) \text { real, } \\
d \Omega & =\left(d \omega_{\mu \nu}\right), \quad d \bar{\Omega}=\left(d \bar{\omega}_{\mu \nu}\right), \\
d Z & =\left(d z_{k l}\right), \quad d \bar{Z}=\left(d \bar{z}_{k l}\right),
\end{aligned}
$$




$$
\begin{aligned}
& \frac{\partial}{\partial \Omega}=\left(\frac{1+\delta_{\mu \nu}}{2} \frac{\partial}{\partial \omega_{\mu \nu}}\right), \quad \frac{\partial}{\partial \bar{\Omega}}=\left(\frac{1+\delta_{\mu \nu}}{2} \frac{\partial}{\partial \bar{\omega}_{\mu \nu}}\right), \\
& \frac{\partial}{\partial Z}=\left(\begin{array}{ccc}
\frac{\partial}{\partial z_{11}} & \cdots & \frac{\partial}{\partial z_{m 1}} \\
\vdots & \ddots & \vdots \\
\frac{\partial}{\partial z_{1 n}} & \cdots & \frac{\partial}{\partial z_{m n}}
\end{array}\right), \quad \frac{\partial}{\partial \bar{Z}}=\left(\begin{array}{ccc}
\frac{\partial}{\partial \bar{z}_{11}} & \cdots & \frac{\partial}{\partial \bar{z}_{m 1}} \\
\vdots & \ddots & \vdots \\
\frac{\partial}{\partial \bar{z}_{1 n}} & \cdots & \frac{\partial}{\partial \bar{z}_{m n}}
\end{array}\right),
\end{aligned}
$$

where $\delta_{i j}$ denotes the Kronecker delta symbol.

C. L. Siegel [17] introduced the symplectic metric $d s_{n}^{2}$ on $\mathbb{H}_{n}$ invariant under the action $(9.1)$ of $S p(n, \mathbb{R})$ given by

$$
d s_{n}^{2}=\sigma\left(Y^{-1} d \Omega Y^{-1} d \bar{\Omega}\right)
$$

and H. Maass [11] proved that the differential operator

$$
\Delta_{n}=4 \sigma\left(Y^{t}\left(Y \frac{\partial}{\partial \bar{\Omega}}\right) \frac{\partial}{\partial \Omega}\right)
$$

is the Laplacian of $\mathbb{H}_{n}$ for the symplectic metric $d s_{n}^{2}$. Here $\sigma(A)$ denotes the trace of a square matrix $A$. In [23], the author proved that for any two positive real numbers $A$ and $B$, the following metric

$$
\begin{aligned}
& d s_{n, m ; A, B}^{2}=A \sigma\left(Y^{-1} d \Omega Y^{-1} d \bar{\Omega}\right) \\
& +B\left\{\sigma\left(Y^{-1 t} V V Y^{-1} d \Omega Y^{-1} d \bar{\Omega}\right)+\sigma\left(Y^{-1 t}(d Z) d \bar{Z}\right)\right. \\
& \left.-\sigma\left(V Y^{-1} d \Omega Y^{-1 t}(d \bar{Z})\right)-\sigma\left(V Y^{-1} d \bar{\Omega} Y^{-1 t}(d Z)\right)\right\}
\end{aligned}
$$

is a Riemannian metric on $\mathbb{H}_{n, m}$ which is invariant under the action (9.2) of the Jacobi group $G_{n, m}^{J}$.

The author [23] proved that for any two positive real numbers $A$ and $B$, the Laplacian $\Delta_{n, m ; A, B}$ of $\left(\mathbb{H}_{n, m}, d s_{n, m ; A, B}^{2}\right)$ is given by

$$
\begin{aligned}
\Delta_{n, m ; A, B}=\frac{4}{A} & \left\{\sigma\left(Y^{t}\left(Y \frac{\partial}{\partial \bar{\Omega}}\right) \frac{\partial}{\partial \Omega}\right)+\sigma\left(V Y^{-1}{ }^{t} V\left(Y \frac{\partial}{\partial \bar{Z}}\right) \frac{\partial}{\partial Z}\right)\right. \\
& \left.+\sigma\left(V^{t}\left(Y \frac{\partial}{\partial \bar{\Omega}}\right) \frac{\partial}{\partial Z}\right)+\sigma\left({ }^{t} V\left(Y \frac{\partial}{\partial \bar{Z}}\right) \frac{\partial}{\partial \Omega}\right)\right\} \\
& +\frac{4}{B} \sigma\left(Y \frac{\partial^{t}}{\partial Z}\left(\frac{\partial}{\partial \bar{Z}}\right)\right) .
\end{aligned}
$$


Using $G_{n, m}^{J}$-invariant differential operators on the Siegel-Jacobi space $\mathbb{H}_{n, m}$, we introduce a notion of Maass-Jacobi forms.

Definition 9.1. Let

$$
\Gamma_{n, m}:=S p(n, \mathbb{Z}) \ltimes H_{\mathbb{Z}}^{(n, m)}
$$

be the discrete subgroup of $G^{J}$, where

$$
H_{\mathbb{Z}}^{(n, m)}=\left\{(\lambda, \mu ; \kappa) \in H_{\mathbb{R}}^{(n, m)} \mid \lambda, \mu, \kappa \text { are integral }\right\} .
$$

A smooth function $f: \mathbb{H}_{n, m} \longrightarrow \mathbb{C}$ is called a Maass-Jacobi form on $\mathbb{H}_{n, m}$ if $f$ satisfies the following conditions (MJ1)-(MJ3) :

(MJ1) $f$ is invariant under $\Gamma_{n, m}$.

(MJ2) $f$ is an eigenfunction of the Laplacian $\Delta_{n, m ; A, B}$ (cf. (9.6)).

(MJ3) $f$ has a polynomial growth, that is, there exist a constant $C>0$ and a positive integer $N$ such that

$$
|f(X+i Y, Z)| \leq C|p(Y)|^{N} \quad \text { as } \operatorname{det} Y \longrightarrow \infty,
$$

where $p(Y)$ is a polynomial in $Y=\left(y_{i j}\right)$.

Remark 9.1. Let $\mathbb{D}_{*}$ be a commutative subalgebra of $\mathbb{D}\left(\mathbb{H}_{n, m}\right)$ containing the Laplacian $\Delta_{n, m ; A, B}$. We say that a smooth function $f: \mathbb{H}_{n, m} \longrightarrow \mathbb{C}$ is a MaassJacobi form with respect to $\mathbb{D}_{*}$ if $f$ satisfies the conditions $(M J 1),(M J 2)_{*}$ and $(M J 3)$ : the condition $(M J 2)_{*}$ is given by

$(M J 2)_{*} f$ is an eigenfunction of any invariant differential operator in $\mathbb{D}_{*}$.

Let $\rho$ be a rational representation of $G L(n, \mathbb{C})$ on a finite dimensional complex vector space $V_{\rho}$. Let $\mathcal{M} \in \mathbb{R}^{(m, m)}$ be a symmetric half-integral semi-positive definite matrix of degree $m$. Let $C^{\infty}\left(\mathbb{H}_{n, m}, V_{\rho}\right)$ be the algebra of all $C^{\infty}$ functions on $\mathbb{H}_{n, m}$ with values in $V_{\rho}$. Let $J_{\rho, \mathcal{M}}: G_{n, m}^{J} \times \mathbb{H}_{n, m} \longrightarrow G L\left(V_{\rho}\right)$ be the canonical automorphic factor for $G_{n, m}^{J}$ on $\mathbb{H}_{n, m}$ given by

$$
\begin{aligned}
J_{\rho, \mathcal{M}}(g,(\Omega, Z))= & e^{2 \pi i \operatorname{tr}\left(\mathcal{M}[Z+\lambda \Omega+\mu](C \Omega+D)^{-1} C\right)} \\
& \times e^{-2 \pi i \operatorname{tr}\left(\mathcal{M}\left(\lambda \Omega^{t} \lambda+2 \lambda^{t} Z+\kappa+\mu^{t} \lambda\right)\right)} \rho(C \Omega+D),
\end{aligned}
$$

where $g=(M,(\lambda, \mu ; \kappa)) \in G_{n . m}^{J}$ with $M=\left(\begin{array}{ll}A & B \\ C & D\end{array}\right) \in S p(n, \mathbb{R})$ and $(\lambda, \mu ; \kappa) \in$ $H_{\mathbb{R}}^{(n, m)}$. We recall the Siegel's notation $\alpha[\beta]={ }^{t} \beta \alpha \beta$ for suitable matrices $\alpha$ and $\beta$.

We define the $\left.\right|_{\rho, \mathcal{M}}$-slash action of $G_{n, m}^{J}$ on $C^{\infty}\left(\mathbb{H}_{n, m}, V_{\rho}\right)$ as follows: If $f \in$ $C^{\infty}\left(\mathbb{H}_{n, m}, V_{\rho}\right)$ and $g \in G_{n, m}^{J}$,

$$
\left(\left.f\right|_{\rho, \mathcal{M}}[g]\right)(\Omega, Z):=J_{\rho, \mathcal{M}}(g,(\Omega, Z))^{-1} f(g \cdot(\Omega, Z)) .
$$


We define $\mathbb{D}_{\rho, \mathcal{M}}$ to be the algebra of all differential operators $D$ on $\mathbb{H}_{n, m}$ satisfying the following condition

$$
\left.(D f)\right|_{\rho, \mathcal{M}}[g]=D\left(\left.f\right|_{\rho, \mathcal{M}}[g]\right)
$$

for all $f \in C^{\infty}\left(\mathbb{H}_{n, m}, V_{\rho}\right)$ and for all $g \in G_{n, m}^{J}$. We denote by $Z_{\rho, \mathcal{M}}$ the center of $\mathbb{D}_{\rho, \mathcal{M}}$.

We define an another notion of Maass-Jacobi forms as follows.

Definition 9.2. A vector-valued smooth function $\phi: \mathbb{H}_{n, m} \longrightarrow V_{\rho}$ is called a Maass-Jacobi form on $\mathbb{H}_{n, m}$ of type $\rho$ and index $\mathcal{M}$ if it satisfies the following conditions $(M J 1)_{\rho, \mathcal{M}},(M J 2)_{\rho, \mathcal{M}}$ and $(M J 3)_{\rho, \mathcal{M}}$ :

$\left.(M J 1)_{\rho, \mathcal{M}} \quad \phi\right|_{\rho, \mathcal{M}}[\gamma]=\phi$ for all $\gamma \in \Gamma_{n, m}$.

$(M J 2)_{\rho, \mathcal{M}} \quad f$ is an eigenfunction of all differential operators in the center $z_{\rho, \mathcal{M}}$ of $\mathbb{D}_{\rho, \mathcal{M}}$.

$(M J 3)_{\rho, \mathcal{M}} \quad f$ has a growth condition

$$
\phi(\Omega, Z)=O\left(e^{a \operatorname{det} Y} \cdot e^{2 \pi \operatorname{tr}\left(\mathcal{M}[V] Y^{-1}\right)}\right)
$$

as $\operatorname{det} Y \longrightarrow \infty$ for some $a>0$.

The case $n=1, m=1$ and $\rho=\operatorname{det}^{k}(k=0,1,2, \cdots)$ was studied by R. Bendt and R. Schmidt [1], A. Pitale [14] and K. Bringmann and O. Richter [4]. The case $n=1, m=$ arbitrary and $\rho=\operatorname{det}^{k}(k=1,2, \cdots)$ was dealt with by C. Conley and M. Raum [5]. In [5] the authors proved that the center $Z_{\operatorname{det}^{k}, \mathcal{M}}$ of $\mathbb{D}_{\operatorname{det}^{k}, \mathcal{M}}$ is the polynomial algebra with one generator $\mathfrak{C}^{k, \mathcal{M}}$ (cf. Theorem 3.2), the so-called Casimir operator which is a $\left.\right|_{\operatorname{det}^{k}, \mathcal{M}^{-} \text {-slash invariant differential operator of degree }}$ three for the case $n=m=1$ or of degree four for the case $n=1, m \geq 2$. As described in Section 6, Bringmann and Richter [4] considered the Poincaré series $\mathcal{P}_{k, \mathcal{M}, s}^{(n, r)}$ (the case $n=m=1$ ) (cf. (6.7)) that is a harmonic Maass-Jacobi form in the sense of Definition 9.2 and investigated its Fourier expansion and its Fourier coefficients. Here the harmonicity of $\mathcal{P}_{k, \mathcal{M}, s}^{(n, r)}$ means that $\mathrm{e}^{k, \mathcal{M}} \mathcal{P}_{k, \mathcal{M}, s}^{(n, r)}=0$, i.e., $\mathcal{P}_{k, \mathcal{M}, s}^{(n, r)}$ is an eigenfunction of $\mathfrak{C}^{k, \mathcal{M}}$ with zero eigenvalue. Conley and Raum [5] generalized the results in [14] and [4] to the case $n=1$ and $m$ is an arbitrary positive integer.

Remark 9.2. In [3], Bringmann, Conley and Richter proved that the center of the algebra of differential operators invariant under the action of the Jacobi group over a complex quadratic field is generated by two Casimir operators of degree three. They also introduce an analogue of Kohnen's plus space for modular forms of halfintegral weight over $K=\mathbb{Q}(i)$, and provide a lift from it to the space of Jacobi forms over $K$.

Definition 9.3. Let $\rho$ and $\rho^{\prime}$ be two rational representations of $G L(n, \mathbb{C})$ on finite dimensional complex vector spaces $V_{\rho}$ and $V_{\rho}^{\prime}$ respectively. Let $\mathcal{M}$ and $\mathcal{M}^{\prime}$ be two symmetric half-integral semi-positive matrices of degree $m$. A differential operator 
$T: C^{\infty}\left(\mathbb{H}_{n, m}\right) \otimes V_{\rho} \longrightarrow C^{\infty}\left(\mathbb{H}_{n, m}\right) \otimes V_{\rho^{\prime}}$ is covariant from $\left.\right|_{\rho, \mathcal{M}}$ to $\left.\right|_{\rho^{\prime}, \mathcal{M}^{\prime}}$ if $T$ satisfies the following condition

$$
\left.T\left(\left.f\right|_{\rho, \mathcal{M}}[g]\right)=\left.(T f)\right|_{\rho^{\prime}, \mathcal{M}^{\prime}}[g]\right)
$$

for all $f \in C^{\infty}\left(\mathbb{H}_{n, m}\right) \otimes V_{\rho}$ and for all $g \in G_{n, m}^{J}$.

Let $\mathbb{D}\left(\rho, \mathcal{M} ; \rho^{\prime}, \mathcal{M}^{\prime}\right)$ be the space of all covariant differential operators on $\mathbb{H}_{n, m}$ from $\left.\right|_{\rho, \mathcal{M}}$ to $\left.\right|_{\rho^{\prime}, \mathcal{M}^{\prime}}$, and let $\mathbb{D}^{q}\left(\rho, \mathcal{N} ; \rho^{\prime}, \mathcal{M}^{\prime}\right)$ be the space of all covariant differential operators of order $\leq q$ on $\mathbb{H}_{n, m}$ from $\left.\right|_{\rho, \mathcal{M}}$ to $\left.\right|_{\rho^{\prime}, \mathcal{M}^{\prime}}$. When $\rho=\rho^{\prime}$ and $\mathcal{M}=\mathcal{M}^{\prime}$, we refer to such differential operators as $\left.\right|_{\rho, \mathcal{M}}$-invariant, and we write simply $\mathbb{D}_{\rho}, \mathcal{M}$ and $\mathbb{D}_{\rho, \mathcal{M}}^{q}$ instead of $\mathbb{D}(\rho, \mathcal{M} ; \rho, \mathcal{M})$ and $\mathbb{D}^{q}(\rho, \mathcal{M} ; \rho, \mathcal{M})$ respectively.

We present the natural problems.

Problem 1. Find the generators of the algebra $\mathbb{D}_{\rho, \mathcal{M}}$.

Problem 2. Find all the relations among a complete list of generators of $\mathbb{D}_{\rho, \mathcal{M}}$.

Finally we consider the special case that $\rho=\mathbf{1}$ is a trivial representation of $G L(n, \mathbb{C})$ and $\mathcal{M}=0$. Let

$$
T_{n, m}:=S(m, \mathbb{C}) \times \mathbb{C}^{(m, n)}
$$

be the complex vector space of dimension $\frac{n(n+1)}{2}+m n$. We obtain the natural action of $U(n)$ on $T_{n, m}$ given by

$$
h \cdot(\omega, \zeta):=\left(h \omega^{t} h, \zeta^{t} h\right), \quad h \in U(n), \omega \in S(m, \mathbb{C}), \zeta \in \mathbb{C}^{(m, n)} .
$$

We refer to [26] for a precise detail. Then the action (9.11) induces the action $\tau_{n, m}$ of $U(n)$ on the polynomial algebra $\operatorname{Pol}\left(T_{n, m}\right)$ consisting of all polynomial functions on $T_{n, m}$. We denote by $\operatorname{Pol}\left(T_{n, m}\right)^{U(n)}$ the subalgebra of $\operatorname{Pol}\left(T_{n, m}\right)$ invariant under the action $\tau_{n, m}$ of $U(n)$. The we have the so-called Helgason map

$$
\Theta_{n, m}: \operatorname{Pol}\left(T_{n, m}\right)^{U(n)} \longrightarrow \mathbb{D}_{\mathbf{1}, 0}=\mathbb{D}(\mathbf{1}, 0 ; \mathbf{1}, 0)
$$

defined by

$$
\left(\Theta_{n, m}(P) f\right)\left(g K^{J}\right)=\left[P\left(\frac{\partial}{\partial t_{\alpha}}\right) f\left(g \exp \left(\sum_{\alpha=1}^{N_{\star}} t_{\alpha} \eta_{\alpha}\right) K^{J}\right)\right]_{\left(t_{\alpha}\right)=0},
$$

where $N_{\star}=n(n+1)+2 m n,\left\{\eta_{\alpha} \mid 1 \leq \alpha \leq N_{\star}\right\}$ is a basis of $\mathfrak{p}_{n, m}^{J}$ and $P \in$ $\operatorname{Pol}\left(T_{n, m}\right)^{U(n)}$. The map $\Theta_{n, m}$ is a linear bijection but is not multiplicative.

The following natural problems arise.

Problem 3. Find a complete list of explicit generators of $\operatorname{Pol}\left(T_{n, m}\right)^{U(n)}$. 
Problem 4. Find all the relations among a complete list of generators of $\operatorname{Pol}\left(T_{n, m}\right)^{U(n)}$.

Problem 5. Find an easy or effective way to express the images of the above invariant polynomials or generators of $\operatorname{Pol}\left(T_{n, m}\right)^{U(n)}$ under the Helgason map $\Theta_{n, m}$ explicitly.

Recently Problem 3 was solved completely in [9].

\section{References}

[1] R. Berndt and R. Schmidt, Elements of the Representation Theory of the Jacobi Group, Progress in Mathematics, 163, Birkhäuser, Basel, 1998.

[2] W. Borho, Primitive und vollprimitive Ideale in Einhüllenden von $\mathfrak{s o}(5, \mathbb{C})$, J. Algebra, 43(1976), 619-654.

[3] K. Bringmann, C. Conley and O. K. Richter, Jacobi forms over complex quadratic fields via the cubic Casimier operators, preprint.

[4] K. Bringmann and O. K. Richter, Zagier-type dualities and lifting maps for harmonic Maass-Jacobi forms, Advances in Math., 225(2010), 2298-2315.

[5] C. Conley and M. Raum, Harmonic Maass-Jacobi forms of degree 1 with higher rank indices, arXiv:1012.289/v1 [math.NT], 13 Dec 2010.

[6] R. Campoamor-Stursburg and S. G. Low, Virtual copies of semisimple Lie algebras in enveloping algebras of semidirect products and Casimir operators, J. Phys. A: Math. Theor., 42(2009), 065205.

[7] S. Helgason, Groups and geometric analysis, Academic Press, New York (1984).

[8] R. Howe, Perspectives on invariant theory: Schur duality, multiplicity-free actions and beyond, The Schur lectures (1992) (Tel Aviv), Israel Math. Conf. Proceedings, vol. 8 (1995), 1-182.

[9] M. Itoh, H. Ochiai and J.-H. Yang, Invariant differential operators on Siegel-Jacobi space, preprint, 2013.

[10] N. N. Lebedev, Special Functions and their Applications, Dover, New York (1972).

[11] H. Maass, Die Differentialgleichungen in der Theorie der Siegelschen Modulfunktionen, Math. Ann., 126(1953), 44-68.

[12] H. Maass, Siegel modular forms and Dirichlet series, Lecture Notes in Math., vol. 216, Springer-Verlag, Berlin-Heidelberg-New York (1971).

[13] H. Ochiai, A remark on the generators of invariant differential operators on SiegelJacobi space of the smallest size, preprint, 2011.

[14] A. Pitale, Jacobi Maass forms, Abh. Math. Sem. Univ. Hamburg, 79(2009), 87-111.

[15] C. Quesne, Casimir operators of semidirect sum Lie algebras, J. Phys. A: Math. Gen., 21(1988), L321-L324. 
[16] J.-P. Serre, A Course in Arithmetic, Springer-Verlag, Berlin-Heidelberg-New York (1973).

[17] C. L. Siegel, Symplectic Geometry, Amer. J. Math., 65(1943), 1-86; Academic Press, New York and London (1964); Gesammelte Abhandlungen, no. 41, vol. II, SpringerVerlag (1966), 274-359.

[18] N.-P. Skoruppa, Explicit formulas for the Fourier coefficients of Jacobi and elliptic modular forms, Invent. Math., 102(1990), 501-520.

[19] G. N. Watson, The Treatise on the Theory of Bessel Functions, Cambridge Univ. Press, London (1962).

[20] J.-H. Yang, The Method of Orbits for Real Lie Groups, Kyungpook Math. J., 42(2)(2002), 199-272 or arXiv:math.RT/0602056.

[21] J.-H. Yang, A note on Maass-Jacobi forms, Kyungpook Math. J. 43(4)(2003), 547566 or arXiv:math.NT/0612387.

[22] J.-H. Yang, A note on a fundamental domain for Siegel-Jacobi space, Houston J. Math., 32(3)(2006), 701-712 or arXiv:math.NT/0507218.

[23] J.-H. Yang, Invariant metrics and Laplacians on Siegel-Jacobi space, Journal of Number Theory, 127(2007), 83-102 or arXiv:math.NT/0507215.

[24] J.-H. Yang, A partial Cayley transform for Siegel-Jacobi disk, J. Korean Math. Soc., 45(2008), 781-794 or arXiv:math.NT/0507216.

[25] J.-H. Yang, Invariant metrics and Laplacians on Siegel-Jacobi disk, Chinese Annals of Mathematics, 31B(1)(2010), 85-100 or arXiv:math.NT/0507217.

[26] J.-H. Yang, Invariant Differential Operators on the Siegel-Jacobi Space, arXiv: 1107.0509v1 [math.NT], 4 July 2011. 